\title{
L'égalitarisme paysan dans l'ancienne société rurale de la vallée du Saint-Laurent : éléments pour une ré-interprétation
}

\section{Christian Dessureault}

Volume 40, numéro 3, hiver 1987

URI : https://id.erudit.org/iderudit/304468ar

DOI : https://doi.org/10.7202/304468ar

Aller au sommaire du numéro

\section{Éditeur(s)}

Institut d'histoire de l'Amérique française

\section{ISSN}

0035-2357 (imprimé)

1492-1383 (numérique)

Découvrir la revue

\section{Citer cet article}

Dessureault, C. (1987). L'égalitarisme paysan dans l'ancienne société rurale de la vallée du Saint-Laurent : éléments pour une ré-interprétation. Revue d'histoire de l'Amérique française, 40(3), 373-407.

https://doi.org/10.7202/304468ar

\section{Résumé de l'article}

L'historiographie a beaucoup insisté sur l'accès plutôt libre à la terre de la paysannerie québécoise du $17^{\mathrm{e}}$ jusqu'au début du $19^{\mathrm{e}}$ siècle. Le statut apparemment uniforme de propriétaire de la plupart des paysans suggère la présence d'un groupe social homogène. Au début du $19^{\mathrm{e}}$ siècle, la différenciation de la paysannerie québécoise devient alors, pour les uns, la conséquence d'un renforcement de l'exploitation féodale et d'une crise rurale néo-malthusienne et, pour d'autres, le résultat d'une pénétration du marché dans l'économie paysanne. Sans rejeter totalement la pertinence de l'un ou l'autre de ces modèles pour expliquer l'accentuation des inégalités économiques dans la société paysanne au début $d u 19^{\mathrm{e}}$ siècle, la hiérarchie paysanne constitue l'une des structures immanentes de l'ancienne économie rurale de la vallée du Saint-Laurent. En plus de considérer les questions primordiales des rapports au marché et à la propriété des paysans, cet article s'attarde aux techniques et aux moyens de la production agricole dans l'ancienne économie rurale. Ce sont dans les fondements matériels de l'existence paysanne, dans le procès de travail et dans les rapports à la production, que se révèlent d'abord le degré et la nature de la hiérarchie paysanne. L'étude des niveaux de fortune et du cycle de la vie conjugale confirme ces divisions sociales et économiques au sein de la paysannerie qui ne reposent pas, comme certains historiens l'ont affirmé, sur une simple différenciation d'ordre démographique.
Tous droits réservés @ Institut d'histoire de l'Amérique française, 1987
Ce document est protégé par la loi sur le droit d'auteur. L'utilisation des services d'Érudit (y compris la reproduction) est assujettie à sa politique d'utilisation que vous pouvez consulter en ligne.

https://apropos.erudit.org/fr/usagers/politique-dutilisation/ 


\title{
L'ÉGALITARISME PAYSAN DANS L'ANCIENNE SOCIÉTÉ RURALE DE LA VALLÉE DU SAINT- LAURENT: ÉLÉMENTS POUR UNE RÉ- INTERPRÉTATION ${ }^{1}$
}

\author{
CHRISTIAN DESSUREAULT \\ Département d' histoire \\ Université de Montréal
}

\begin{abstract}
RÉSUMÉ
L'historiographie a beaucoup insisté sur l'accès plutôt libre à la terre de la paysannerie québécoise du $17 \mathrm{e}$ jusqu'au début du $19 \mathrm{e}$ siècle. Le statut apparemment uniforme de propriétaire de la plupart des paysans suggère la présence d'un groupe social homogène. Au début du $19 \mathrm{e}$ siècle, la différenciation de la paysannerie québécoise devient alors, pour les uns, la conséquence d'un renforcement de l'exploitation féodale et d'une crise rurale néo-malthusienne et, pour d'autres, le résultat d'une pénétration du marché dans l'économie paysanne. Sans rejeter totalement la pertinence de l'un ou l'autre de ces modèles pour expliquer l'accentuation des inégalités économiques dans la société paysanne au début du $19 \mathrm{e}$ siècle, la hiérarchie paysanne constitue l'une des structures immanentes de l'ancienne économie rurale de la vallée du Saint-Laurent. En plus de considérer les questions primordiales des rapports au marché et à la propriété des paysans, cet article s'attarde aux techniques et aux moyens de la production agricole dans l'ancienne économie rurale. Ce sont dans les fondements matériels de l'existence paysanne, dans le procès de travail et dans les rapports à la production, que se révèlent d'abord le degré et la nature de la hiérarchie paysanne. L'étude des niveaux de fortune et du cycle de la vie conjugale confirme ces divisions sociales et économiques au sein de la paysannerie qui ne reposent pas, comme certains historiens l'ont affirmé, sur une simple différenciation d'ordre démographique.
\end{abstract}

\section{ABSTRACT}

The litterature concerning pre-industrial Québec has insisted on the peasantry's easy access to land from the seventeenth to the nineteenth century and the apparent uniformity in this group's social status that resulted. Differenciation within the peasantry that appeared at the beginning of the nineteenth century was interpreted, by some, as the consequence of a reinforced feudalism and a neo-malthusian rural crisis or, by others, as the result of a more market oriented peasant economy. Without completely rejecting either of these explanatory models, this article stresses that a hierarchy within the peasantry is an inherent structure of the pre-industrial rural economy of the St. Lawrence valley. As well as considering the peasant's relationship to markets and to land ownership, the technics and means of agricultural production are examined. The degree and nature of social inequality among the peasantry is revealed by looking at the material basis of peasant life, the work processes and the relationship to production. The study of peasant fortunes and the cycle of married life confirm that the social and economic divisions within the peasantry are not only due to demographic differenciation as some authors have maintained.

1 Ce texte présente certaines des conclusions de notre thèse de doctorat, Les fondements de la hiérarchie sociale au sein de la paysannerie. Le cas de Saint-Hyacinthe 1760-1815. Thèse de doctorat manuscrite, Université de Montréal, 1985. Nous tenons à remercier notre directeur de recherche Jean-Pierre Wallot, archiviste fédéral, pour ses conseils judicieux. 


\section{INTRODUCTION}

La société rurale du Québec pré-industriel constitue présentement l'un des champs d'étude privilégiés de l'historiographie. Au cours de la dernière décennie, de nombreuses recherches ont approfondi nos connaissances du régime seigneurial, des rapports marchands dans les campagnes, de la transmission des avoirs familiaux et de la reproduction sociale au sein de la paysannerie. La présente étude s'insère dans ce vaste redéploiement de la recherche historique sur le fonctionnement de l'économie et de la société rurales québécoises pré-industrielles. Elle privilégie plus spécifiquement l'analyse de la hiérarchisation au sein de . la paysannerie.

L'étude de la hiérarchie paysanne apparaît essentielle à une meilleure compréhension de la société québécoise d'avant 1850. Jusqu'à présent, les études sur les structures sociales en Nouvelle-France et au Bas-Canada ont adopté une approche macroscopique de la réalité sociale $^{2}$. Notre propos n'est pas de juger ici de la valeur et de la pertinence des divers modèles de stratification sociale proposés ${ }^{3}$. Il convient toutefois de souligner que ces découpages par le haut de l'espace social ont davantage ciselé les contours des classes ou des strates supérieures, lieux de pouvoirs économique et politique, au détriment de la base même de l'édifice de la société bas-canadienne: la paysannerie. A cet égard, une approche microscopique ${ }^{4}$ centrée sur la société paysanne permettrait de mieux définir cette masse rurale qui regroupe certainement près des trois quarts de la population bas-canadienne au tournant du 19 e siècle 5 .

2 L'approche macroscopique consiste à repérer «les agrégats (sociaux) et leurs réflexes collectifs sans les dériver de leurs unités particulières». G. Paquet et J.-P. Wallot, «Groupes sociaux et pouvoir: le cas bas-canadien au tournant du XIXe siècle», Revue d'histoire de l'Amérique française, 27,4 (mars 1974): 525.

3 Pour des modèles de stratification sociale proposés à partir d'une approche plutôt macroscopique de la réalité sociale bas-canadienne, voir G. Bernier, «La structure de classes québécoise au $19 \mathrm{e}$ siècle et le problème de l'articulation des modes de production", Revue canadienne de science politique, 14,3 (septembre 1981): 487-518; G. Bourque et N. Laurin-Frenette, «La structure nationale québécoise», Socialisme québécois, 5,21-22 (1971): 109-155; A. Dubuc, «Problems in the Study of Stratification of the Canadian Society from 1760 to 1840», Canadian Historical Association Annual Report (1965): 13-29; G. Paquet et J.-P. Wallot, loc. cit., 509554.

4 Dans un premier temps, l'approche macroscopique permet d'identifier les principaux groupes sociaux correspondant aux diverses pratiques économiques et politiques, ainsi qu'aux grandes articulations de l'action collective. Cependant, ce découpage théorique de la réalité sociale doit être confronté aux résultats de travaux plus approfondis, segment par segment, du système économique et social. Sur la nécessité d'une démarche interactive de la recherche historique, voir G. Paquet et J.-P. Wallot, «Pour une méso-histoire du XIXe siècle canadien», RHAF, 33,3 (décembre 1979): 387-425.

5 G. Paquet et J.-P. Wallot, «Groupes sociaux...», loc. cit., 546-547. Malheureusement, nous ne disposons pas de données précises sur la composition socio-professionnelle de la population bas-canadienne au tournant du 19e siècle. Nous nous référons donc aux approximations des professeurs Paquet et Wallot. Il s'agit simplement de souligner l'importance démographique de ceux qu'on nomme tantôt les habitants, tantôt les paysans, tantôt les censitaires-paysans, tantôt les classes populaires rurales. 


\section{I - BILAN HISTORIOGRAPHIQUE}

Pendant longtemps, l'historiographie a entretenu le mythe d'une paysannerie canadienne-française plutôt homogène du $17 \mathrm{e}$ au milieu du 19 e siècle $^{6}$. Au cours de la phase initiale de la formation d'une société rurale dans la vallée du Saint-Laurent, l'accès large à la propriété foncière, la faiblesse des prélèvements extérieurs sur la production agricole (les dîmes et les rentes seigneuriales), ainsi qu'une rationalité paysanne axée d'abord sur l'auto-subsistance, ont conditionné le maintien d'une société paysanne relativement égalitaire, composée majoritairement de petits propriétaires plutôt pauvres mais indépendants des pressions extérieures, tant seigneuriales que marchandes ${ }^{7}$.

A partir de ces constats explicites ou implicites des historiens, Denis Monière propose pour la Nouvelle-France l'existence d'un mode spécifique de petits producteurs regroupant des artisans et surtout des paysans ${ }^{8}$. Ce mode de production, quoique soumis au capitalisme marchand dominant dans la formation sociale, aurait évolué selon ses propres règles de fonctionnement. Selon $\mathrm{D}$. Monière, la Conquête aurait eu pour effet majeur de généraliser à l'ensemble de la structure sociale canadienne-française ce système économique «privé d'antagonismes de classes» ${ }^{9}$.

D'autres auteurs marxistes québécois rejettent ce modèle des petits producteurs et insistent plutôt sur la nette domination du féodalisme dans les campagnes, voire dans l'ensemble de la formation sociale de la Nouvelle-France et du Bas-Canada ${ }^{10}$. Néanmoins, la paysannerie québécoise, tantôt soumise, tantôt révoltée contre les exactions féodales, leur apparaît comme un groupe social homogène: des censitaires

6 A cet égard, la société paysanne, définie par Maurice Séguin dans La Nation canadienne et l'agriculture, constitue le modèle le plus articulé de la paysannerie canadienne-française comme groupe social homogène. M. Séguin, La Nation «canadienne» et l'agriculture (1765-1850) (TroisRivières, Boréal Express, 1970).

7 Pour une analyse des divers courants historiographiques qui reprennent tour à tour cette conception de la société rurale de la vallée du Saint-Laurent du 17e au début du $19 \mathrm{e}$ siècle, voir F. Ouellet, «La formation d'une société dans la vallée du Saint-Laurent: d'une société sans classe à une société de classes», Canadian Historical Review, 62,4 (1981): 407-450.

8 D. Monière, «L'utilité du concept de mode de production des petits producteurs pour l'historiographie de la Nouvelle-France», $R H A F, 29,4$ (mars 1976): 481-501; dès 1974, Marcel Rioux et ses collaborateurs avaient déjà esquissé la thèse d'un mode spécifique de production des petits producteurs dans le Québec rural pré-industriel dont ils limitaient l'application à l'aprèsConquête, le considérant d'ailleurs comme le résultat de cette dernière. Y. Lamarche, M. Rioux et R. Sévigny, Aliénation et idéologie dans la vie quotidienne des Montréalais francophones (Montréal, Presses de l'Université de Montréal, 1974).

9 Ibid., 500.

10 Lise Pilon-Lê, «Le régime seigneurial au Québec: contribution à une analyse de la transition du féodalisme au capitalisme», Cahiers du socialisme, 6 (automne 1980): 133-168; G. Bourque et A. Légaré, Le Québec. La question nationale (Paris, Maspéro, 1979); G. Bernier, «Sur quelques effets de la rupture structurelle engendrée par la Conquête au Québec, 1760-1854», $R H A F, 35,1$ (juin 1981): 69-95; G. Bernier et D. Salée, "Appropriation foncière et bourgeoisie marchande: éléments pour une analyse de l'économie marchande du Bas-Canada avant 1846», $R H A F, 36,2$ (septembre 1982): 163-194. 
qui se définissent d'abord dans leur opposition de classe aux seigneurs. Dans la première moitié du $19 \mathrm{e}$ siècle, l'accroissement du taux de prélèvement seigneurial et l'alourdissement de l'ensemble des charges du censitaire compromettent progressivement l'accès des paysans à la propriété foncière, surtout aux terres neuves, et rendent désormais incertaine la reproduction simple de la paysannerie. D'après leur modèle classique de la transition du féodalisme au capitalisme, la différenciation sociale de la paysannerie s'enclenche alors graduellement sous la pression d'une sur-exploitation féodale et le mécanisme conduit inévitablement à la dépossession de la paysannerie: préalable, selon eux, au développement du capitalisme ${ }^{11}$.

De fait, à défaut de recherches empiriques fouillées, ces modèles marxistes de la transition du féodalisme au capitalisme reprennent dans ses grandes lignes, mais de manière moins prudente et moins nuancée, la trame historique de Fernand Ouellet sur la crise structurelle de la société rurale bas-canadienne dans la première moitié du $19 \mathrm{e}$ siècle ${ }^{12}$. Selon F. Ouellet, la différenciation sociale au sein de la paysannerie québécoise s'amorce véritablement au début du $19 \mathrm{e}$ siècle. Elle est la conséquence directe d'une crise agricole majeure et le résultat, à plus long terme, d'un blocage malthusien dans la vallée du Saint-Laurent: c'est-à-dire d'une trop forte pression démographique par rapport aux terres disponibles. Cependant, la politique foncière des seigneurs et, dans une moindre mesure, celle des spéculateurs dans les cantons ont accéléré et amplifié les mécanismes de ce blocage malthusien. La crise rurale se concrétise par l'appauvrissement généralisé de la paysannerie et par son repliement vers une agriculture d'auto-subsistance. Le manque de terres et les pratiques successorales provoquent le morcellement

11 Nous qualifions ce modèle de classique en ce sens qu'il se réfère à la lettre aux conclusions de K. Marx sur les rapports étroits entre l'expropriation de la paysannerie et la formation du prolétariat en Angleterre. Depuis déjà plusieurs années, les historiens marxistes ont fortement nuancé, sinon rejeté, la portée universelle du cas anglais comme voie de passage du féodalisme au capitalisme. M. Dobbs et P.-M. Sweezy, Du féodalisme au capitalisme: problème de la transition (Paris, Maspéro, 1977); F. Gauthier, La voie paysanne dans la Révolution française. L'exemple Picard (Paris, Maspéro, 1977). Par ailleurs, les fondements historiques de la thèse marxiste de l'expulsion de la yeomanry et de la dépopulation des campagnes anglaises avant 1850 sont euxmêmes remis en question dans l'historiographie britannique. A cet égard, voir C. Couture, «La Conquête de 1760 et le problème de la transition au capitalisme», $R H A F, 39,3$ (hiver 1986): 369389.

12 La crise de la société rurale bas-canadienne constitue l'un des principaux aspects historiographiques de l'oeuvre de Fernand Ouellet. Dans un premier temps, en collaboration avec Jean Hamelin, il développe la thèse d'une crise agricole majeure au Bas-Canada de la première décennie jusqu'au milieu du 19e siècle. Les pratiques agricoles déficientes et arriérées des paysans sont l'une des principales causes du déclenchement de cette crise agricole. J. Hamelin et F. Ouellet, «La crise agricole dans le Bas-Canada, 1802-1837», Études rurales, 3,4 (1962-1963): 35-57. Par la suite, F. Ouellet élargit et affine la thèse de la crise agricole qui devient de plus en plus celle d'une crise structurelle globale de la société rurale bas-canadienne reposant sur diverses causes (mauvaises pratiques agricoles, épuisement et morcellement des terres, accroissement des charges seigneuriales) avec, comme toile de fond, la rareté des terres neuves et la trop forte pression démographique. F. Ouellet, Le Bas-Canada 1791-1840. Changements structuraux et crise (Ottawa, Éditions de l'Université d'Ottawa, 1980). 
des propriétés foncières. La combinaison de ces facteurs accélère, selon l'auteur, la formation d'un prolétariat rural. De la masse paysanne se démarque toutefois une élite composée principalement de cultivateurs d'origine britannique ou américaine dont la production agricole est orientée en fonction du marché ${ }^{13}$. Seuls quelques cultivateurs canadiens-français réussissent à imiter avec succès leurs compatriotes anglosaxons.

Dans un ouvrage récent, Allan Greer reprend partiellement ce modèle néo-malthusien de la différenciation sociale au sein de la paysannerie québécoise pré-capitaliste ${ }^{14}$. Jusqu'au début du $19 \mathrm{e}$ siècle, cette paysannerie se définit, selon l'auteur, comme un groupe social particulièrement homogène. Dans la première moitié du $19 \mathrm{e}$ siècle, la composition de la société paysanne se diversifie. On assiste alors à la formation, à côté du groupe fortement majoritaire des propriétaires-exploitants, d'un second groupe plutôt marginal de fermiers-métayers, et d'un dernier groupe de plus en plus important de journaliers agricoles. D'après A. Greer, cette différenciation tardive de la société paysanne n'est pas le produit, comme en Europe, de la pénétration et du développement du capitalisme marchand dans les campagnes. L'auteur l'attribue plutôt aux effets conjugués de la trop forte pression démographique et de la rareté croissante des terres neuves. De plus, il invoque les arguments du cycle de la vie familiale pour réfuter l'existence de divisions sociales au sein de la paysannerie. A l'instar du modèle néo-marxiste américain de la Household Economy ${ }^{15}$, l'accumulation et la concentration des richesses lui apparaissent contraires à la rationalité d'une économie paysanne d'abord axée sur l'auto-subsistance et sur la reproduction du groupe familial.

Par ailleurs, Gilles Paquet et Jean-Pierre Wallot associent plus volontiers la dynamique de la différenciation sociale dans les campagnes bas-canadiennes à une intégration de plus en plus poussée de

13 Sur ces aspects du clivage socio-ethnique au sein de la paysannerie bas-canadienne, voir F. Ouellet, «Le clivage socio-ethnique, la structure sociale et la mise en valeur du terroir dans les seigneuries de La Salle et de Châteauguay en 1831», Éléments d'histoire sociale du Bas-Canada (Montréal, Éditions Hurtubise HMH, 1972), 153-173; comme l'a très bien fait ressortir Pierre Tousignant, les travaux de F. Ouellet sont profondément marqués par une conception néo-wébérienne de la structure sociale. En effet, cet historien adopte «une typologie différentielle des mentalités» selon l'appartenance ethnique à l'intérieur de chaque groupe ou de chaque agrégat social: paysans, marchands, seigneurs. P. Tousignant, «Le Bas-Canada 1791-1840: une étape importante dans l'oeuvre de Fernand Ouellet», RHAF, 34,4 (juin 1981): 415-436.

${ }_{14}$ A. Greer, Peasant, Lord and Merchant. Rural Society in Three Quebec Parishes 17401840 (Toronto, University of Toronto Press, 1985).

15 Pour les tenants de la Household Economy, voir C. Clark, «Household Economy, Market Exchange and the Aim of Capitalism in the Connecticut Valley, 1800-1860», Journal of Social History, 13 (1979): 168-169; J. A. Henretta, «Families and Farms: Mentalité in Pre-Industrial America», William and Mary Quarterly, 35,1 (January 1978): 3-32; M. Merril, «Cash is Good to Eat: Self-Sufficiency and Exchange in the Rural Economy of the United States», Radical History Review, 20 (Winter 1977): 42-72. 
l'économie paysanne à une économie de marché ${ }^{16}$. Grâce aux inventaires après décès, leur analyse des niveaux de fortune des paysans ne se limite plus au seul indicateur foncier ${ }^{17}$. Elle englobe désormais l'ensemble du discours de la consommation et de la production. Or, tant du côté des facteurs de production que de celui des biens de consommation, G. Paquet et J.-P. Wallot notent un accroissement sensible de la richesse des ménages paysans bas-canadiens au début du 19e siècle. Cet accroissement de la richesse ne se répartit toutefois pas uniformément entre les habitants. Selon ces auteurs, la différenciation sociale que sous-tend ce processus de redistribution inégale des biens mobiliers et immobiliers, s'inscrit dans un procès plus large de modernisation de l'ensemble des structures économiques et sociales au Bas-Canada. A l'instar de la plupart des historiens ${ }^{18}$, la hiérarchie paysanne ne leur apparaît pas comme l'une des structures immanentes de l'ancienne société rurale de la vallée du Saint-Laurent, inscrite dans le fonctionnement même de l'économie rurale et antérieure au procès de modernisation qu'ils ont repéré.

\section{2 - TERROIR DE PEUPLEMENT ET ÉGALITARISME PAYSAN}

Certes, la division sociale laboureurs-manoeuvriers n'est guère applicable dans le contexte nord-américain. Même en Europe, les hiérarchies paysannes sont rarement aussi tranchées. Mais au-delà des nombreuses nuances propres à chaque pays, à chaque région, à chaque terroir, les inégalités de fortune, de pouvoir et de prestige au sein des sociétés paysannes pré-capitalistes dont l'économie repose sur une polyculture à dominante céréalière ne s'exprimeraient-elles pas, selon l'ancien modèle proposé par Marc Bloch, dans la possession ou le manque d'attelage pour les travaux de culture ${ }^{19}$ ?

En Amérique du Nord, cette division typique d'Ancien Régime aurait fort bien pu être occultée par le statut apparemment uniforme de

16 G. Paquet et J.-P. Wallot, «Crise agricole et tensions socio-ethniques dans le Bas-Canada 1802-1812: éléments pour une ré-interprétation», $R H A F, 26,2$ (septembre 1972): 185-237.

${ }_{17}$ Idem, «Structures sociales et niveaux de richesse dans les campagnes du Québec, 17921812», Bulletin d' histoire de la culture matérielle, 13 (printemps 1983): 25-44.

18 Dans Économie et société en Nouvelle-France, Jean Hamelin avait déjà souligné les inégalités économiques et sociales de la paysannerie canadienne et décrit les conséquences éventuelles de celles-ci lors des crises cycliques de production en Nouvelle-France. Cependant, J. Hamelin ne s'est pas penché sur l'analyse même de cette hiérarchie paysanne, de ses fondements et de sa nature (J. Hamelin, Économie et société en Nouvelle-France (Québec, Presses de l'Université Laval, 1960), 65-71). Dans des recherches menées sur Varennes et Verchères, Louis Michel soulève égalemerit le problème de la hiérarchie paysanne et ses conséquences sur la transmission des avoirs familiaux et sur la reproduction sociale au sein de la paysannerie (L. Michel, «Varennes et Verchères, des origines au milieu du XIXe siècle: état d'une enquête» dans J. Goy et J.-P. Wallot, éd., Évolution et éclatement du Monde rural. France-Québec XVIIe-XXe siècles (Montréal, Presses de l'Université de Montréal, 1986), 325-340).

${ }_{19} \mathrm{M}$. Bloch, Les caractères originaux de l' histoire rurale française (Paris, Armand Colin, 1955-1956), 194. 
propriétaire-exploitant de la plupart des paysans, surtout dans les nouveaux terroirs de peuplement. Longtemps, la propriété foncière est apparue d'autant plus accessible à la masse rurale que la terre demeurait abondante et qu'elle n'acquérait une forte valeur marchande ${ }^{20}$ qu'au fur et à mesure du labeur des colons. D'après les modèles frontiéristes et néo-frontiéristes de l'historiographie nord-américaine, les nouvelles communautés rurales apparaissaient alors comme des exemples d'égalitarisme paysan ${ }^{21}$.

En général, l'historiographie a beaucoup insisté sur l'accès plutôt large à la terre de la paysannerie québécoise du 17e jusqu'au début du $19 \mathrm{e}$ siècle, voire jusqu'en 1815 . Néanmoins, sans négliger cette question fondamentale des rapports à la propriété, il faut désormais s'attarder aux méthodes et aux techniques de la production agricole dans l'ancienne économie rurale pour déterminer les conditions réelles dans lesquelles s'exerce le travail paysan. Ce sont dans les fondements matériels de l'existence paysanne, dans le procès de travail et dans les rapports à la production, que nous pouvons saisir le degré et la nature de la hiérarchie paysanne.

Nous avons voulu revoir cette question de la hiérarchie paysanne dans le cadre d'un terroir de peuplement récent de la région de Montréal au tournant du 19e siècle: la seigneurie de Saint-Hyacinthe (voir carte 1). La frontière chronologique de 1815 s'est rapidement imposée. La phase initiale de la formation et de l'organisation d'une nouvelle société rurale apparaît alors vraiment terminée. L'économie rurale maskoutaine a atteint un seuil de productivité comparable à celui des vieux terroirs de la vallée du Saint-Laurent. Bon an mal an, la région fournit alors des excédents de céréales ${ }^{22}$. Au début du $19 \mathrm{e}$ siècle, les paysans maskoutains ont dû affronter leurs premières graves difficultés économiques $^{23}$ et, en 1815 , ils sont à l'aube d'une série de mauvaises

20 Cela n'implique pas nécessairement l'absence de spéculation sur les terres neuves, mais seulement que celles-ci demeuraient tout de même accessibles à la plupart des paysans, même aux plus pauvres d'entre eux.

21 Pour une critique du mythe égalitariste de la société américaine à l'époque coloniale, voir G. L. Main, «Inequality in Early America: the Evidence From Probate Records of Massachussets and Maryland», The Journal of Interdisciplinary History, 7,4 (Spring 1977): 559-582.

22 Par exemple, lors de la récolte désastreuse de 1815 , la plaine maskoutaine est l'une des rares régions excédentaires du Bas-Canada. Voir $J C A B C, 1816$, appendice I.

${ }_{23}$ Certains indices viennent appuyer l'hypothèse d'une grave crise ponctuelle de la production agricole dans la plaine maskoutaine pour les récoltes de 1802 et de 1803 . Notons d'abord une hausse spectaculaire de l'endettement notarié des paysans maskoutains en 1803 et en 1804 . La majeure partie des obligations consenties par les paysans au cours de ces deux années constituent alors des consolidations de dettes pour consommation auprès des marchands locaux et forains, qui, eux-mêmes, doivent subir le même sort en raison des rappels de créances de leurs fournisseurs. Par ailleurs, la forte hausse des mortalités infantiles et juvéniles en 1803 et 1804 n'est probablement pas tout à fait étrangère à la mauvaise conjoncture économique. Auparavant, les premiers colons maskoutains avaient également connu deux autres crises ponctuelles de production d'ancien type apparemment assez aiguës, soit en 1784 et en 1789 . C. Dessureault, Les fondements de la hiérarchie sociale au sein de la paysannerie. Le cas de Saint-Hyacinthe 1760-1815. Thèse de doctorat manuscrite, Université de Montréal, 1985. 


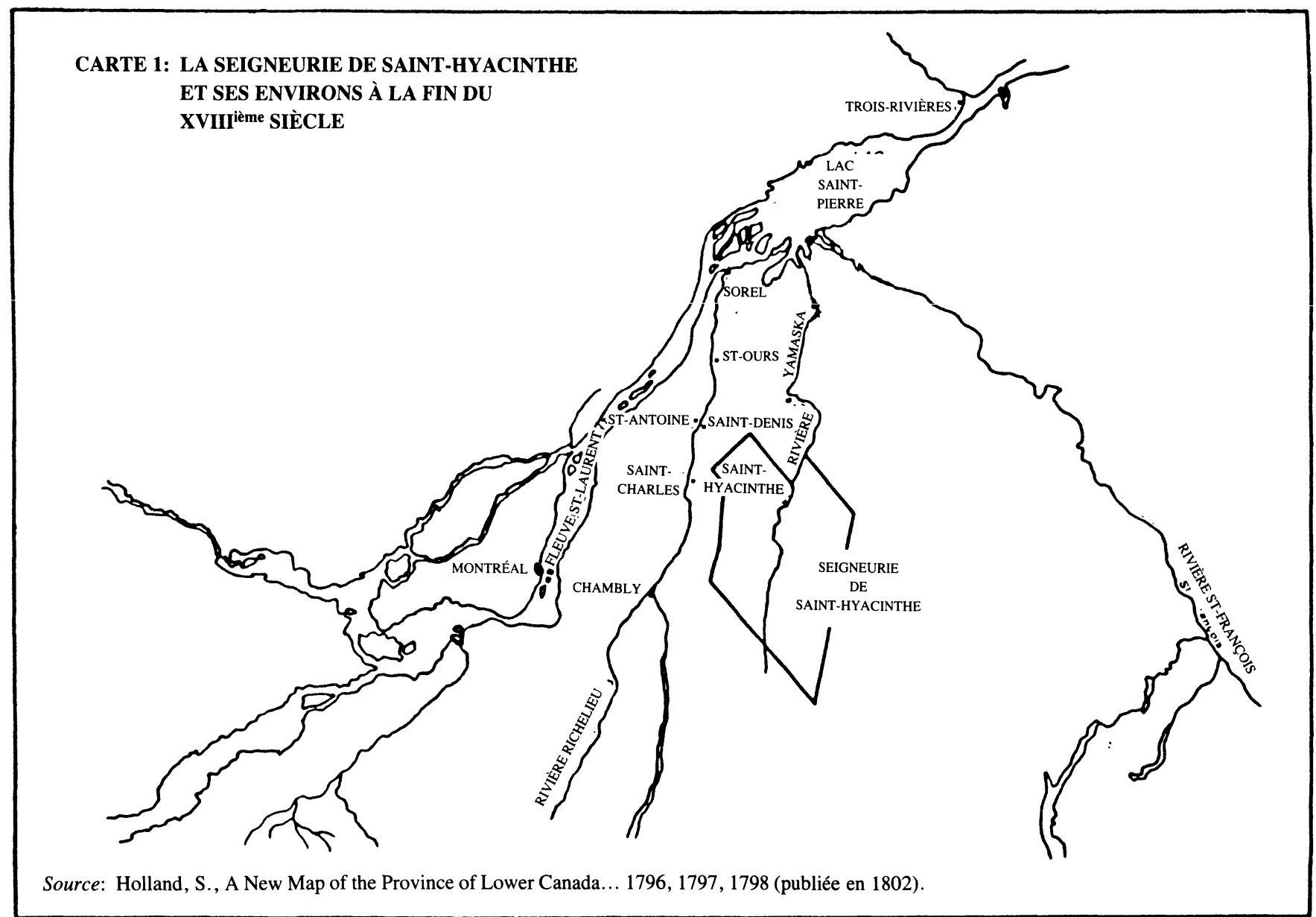

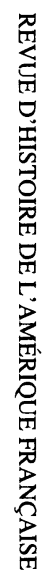


récoltes successives qui risquent de perturber et de modifier durablement l'ancienne hiérarchie paysanne. Par ailleurs, la masse des documents notariés et la lourdeur d'une exploitation sérielle et systématique des inventaires après décès commandaient aussi cette limite chronologique.

\section{3 - ÉCONOMIE PAYSANNE: AUTO-CONSOMMATION ET MARCHÉ}

Certains historiens lient l'apparition de divisions sociales au sein de la paysannerie québécoise au développement du capitalisme marchand dans les campagnes durant la première moitié du $19 \mathrm{e}$ siècle. Auparavant, les exploitations paysannes étaient, selon eux, absentes voire réfractaires à l'économie de marché parce qu'elles demeuraient toujours soumises aux dictats de l'auto-consommation familiale. Il apparaît donc nécessaire de discuter cette vision dichotomique de la place respective de l'auto-consommation et du marché dans l'économie paysanne pré-capitaliste.

La notion d'économie paysanne, comme système de production, nous est apparue la plus appropriée pour saisir la dynamique de l'agriculture maskoutaine de la fin du $18 \mathrm{e}$ et du début du $19 \mathrm{e}$ siècle ${ }^{24}$. La cellule de base de ce système de production est l'exploitation familiale. La production agricole est presque entièrement réalisée au sein de petites unités relativement autonomes de production et de consommation nommés groupes domestiques qui, dans le cas maskoutain, s'assimilent majoritairement aux familles conjugales.

L'un des objectifs prioritaires des familles paysannes est assurément de répondre, à même leurs propres ressources, à leurs principaux besoins domestiques: en particulier d'assurer la subsistance et la reproduction du groupe familial. Cependant, ce que certains, comme les tenants de la Household Economy ${ }^{25}$ identifient comme l'idéal-type de

24 La caractérisation de l'économie paysanne comme système spécifique de production a déjà donné lieu à plusieurs modèles dont ceux de $\mathrm{A}$. V. Chayanov, H. Mendras, J. Tepicht et D. Thorner. Parmi ces derniers, nous privilégions certains aspects des modèles de Mendras et de Tepicht. Pour H. Mendras, l'économie paysanne se définit comme un système mi-autarcique, mimarchand, dont la cellule de base est le groupe domestique. Cette économie paysanne doit à la fois répondre à la logique de l'auto-subsistance des membres du G. D. et fournir, sous diverses formes, des prélèvements à l'économie englobante. La rationalité première des paysans est toutefois de répondre d'abord, à même leurs propres ressources, aux besoins fondamentaux de leurs familles, H. Mendras, «Un schéma d'analyse de la paysannerie occidentale», Peasant Studies Newsletters, 1,3-4 (July-October 1972): 78-89, 126-139. Pour J. Tepicht, les rapports entre l'économie paysanne et l'économie englobante revêtent plus d'importance dans le fonctionnement de ce système de production qui n'est ni totalement intégré au marché, ni simplement orienté en fonction des objectifs d'auto-subsistance et de reproduction simple des familles paysannes. De plus, au sein même de la société paysanne, il existe à la fois une forte complémentarité et une opposition virtuelle de classe entre des unités de production économiquement et socialement très différenciées. J. Tepicht, Marxisme et agriculture. Le paysan polonais (Paris, Armand Colin, 1973), 13-46.

C. Clark, loc. cit.; J. A. Henretta, loc. cit.; M. Merril, loc. cit. 
l'auto-consommation, n'empêche aucunement le paysan de produire également pour le marché des surplus commercialisables. L'importance de ces surplus varie selon la taille et la qualité de l'exploitation agricole, selon les besoins domestiques de la famille, selon les variations climatiques et, aussi, selon les opportunités de commercialisation. Par contre, l'objectif de l'auto-subsistance intervient davantage dans la sphère des facteurs de production nécessaires au fonctionnement de l'entreprise paysanne: semences, engrais, animaux de trait, bêtes de reproduction. La rationalité économique paysanne consiste à la fois à vendre le plus d'excédents possibles sur le marché et à réduire au minimum les achats à l'extérieur de l'exploitation. Ce dernier objectif constitue en quelque sorte un frein à la spécialisation de l'exploitation paysanne et à son intégration dans l'économie englobante.

A l'instar de Marcel Aymard ${ }^{26}$ et de Betty Hobbs Pruitt ${ }^{27}$, il nous apparaît illusoire d'opposer irréductiblement l'auto-consommation et la commercialisation dans les sociétés paysannes pré-industrielles. Les plus riches paysans sont souvent ceux qui participent le plus activement à l'économie de marché et qui, en même temps, satisfont le mieux aux objectifs de l'auto-consommation grâce à un éventail très diversifié de production. La vente d'excédents commercialisables permet non seulement à ces derniers d'acquitter facilement leurs divers prélèvements extérieurs comme les dîmes, les droits seigneuriaux et les rentes viagères; à défrayer divers services économiquement ou socialement nécessaires comme ceux des artisans, des médecins et des notaires; à acquérir divers produits manufacturés ou exotiques que ces paysans ne peuvent eux-mêmes produire; mais aussi à augmenter leurs propres moyens de production et à accroître leur niveau de vie et leur prestige social. A l'opposé, nombreux sont les petits exploitants qui n'atteignent pas le seuil de l'auto-subsistance et qui doivent se procurer sur le marché local l'un ou l'autre des produits de base nécessaires à la consommation domestique. En contrepartie, ces petits exploitants doivent vendre, au moins sur une base occasionnelle, leur force de travail.

$\mathrm{Si}$, à long terme, le développement des forces productives dans l'agriculture mène à l'éclatement du cadre de l'auto-consommation et à l'intégration de plus en plus poussée de l'exploitation agricole dans l'économie englobante, la richesse d'un ménage paysan comme son envers la pauvreté, ne se fonde certainement pas sur une mentalité plus ou moins ouverte face au marché tant du côté de la commercialisation des récoltes que de celui des achats de consommation même si ces deux

26 M. Aymard, «Autoconsommation et marchés: Chayanov, Labrousse ou Le Roy Ladurie?», Annales ESC, 38,6 (nov.-déc. 1983): 1392-1410.

B. Hobbs Pruitt, «Self-Sufficiency and the Agricultural Economy of Eighteenth Century Massachusetts», William and Mary Quarterly, 41,3 (July 1984): 333-364. 
dimensions de l'action marchande, conjuguées aux fortes variations du volume des récoltes, favorisent la polarisation de la société paysanne ${ }^{28}$.

La répartition inégale des moyens de production au sein de la paysannerie établit, d'une manière plus ou moins mouvante dans le temps et dans l'espace, une ligne de partage entre les paysans capables d'accéder au marché en position de force comme principaux vendeurs de denrées agricoles, rassembleurs de terre, prêteurs de grains et de numéraire, voire employeurs éventuels, et ceux qui doivent recourir au même marché dans une position plus précaire comme acheteurs de denrées de subsistance, emprunteurs et main-d'oeuvre occasionnelle ou permanente. Les rapports marchands comme les crises de production d'ancien type influent sur l'évolution, à moyen et à long terme, de cette division sociale au sein de la paysannerie; mais ils agissent à l'intérieur et dans la mesure d'une hiérarchie immanente à l'ancienne économie rurale.

\section{4 - L'AGRICULTURE: MÉTHODES ET TECHNIQUES DE PRODUCTION}

Cependant, avant d'analyser la répartition des moyens de production au sein du groupe paysan, il convient de rappeler certains traits du système cultural. Au tournant du $19 \mathrm{e}$ siècle, le blé représente plus des trois quarts des récoltes et des réserves paysannes (voir tableau 1).

Tableau 1

Composition du produit agricole des exploitations paysannes de Saint-Hyacinthe, 1795-1814

(d'après le \% sur la valeur totale des produits)

\begin{tabular}{lcc}
\hline Type de production & $1795-1804$ & $1805-1814$ \\
\hline & $\%$ & $\%$ \\
Avoine & 5,5 & 7,1 \\
Blé & 75,4 & 60,5 \\
Orge & 2,1 & 1,6 \\
Autres céréales & 0,1 & 0,6 \\
Pois & 11,9 & 12,0 \\
Pommes de terre & - & 4,9 \\
Lin & 0,2 & 0,6 \\
Légumes-tabac & 0,3 & 0,2 \\
Fourrages & 4,5 & 12,5 \\
TOTAL & 100,0 & 100,0 \\
\hline
\end{tabular}

Source: Inventaires après décès.

28 A ce sujet, voir L. Michel, «Endettement et société rurale dans la région de Montréal au dix-huitième siècle. Premières approches et éléments de réflexion». Communication présentée au colloque d'histoire comparée des sociétés rurales de la France et du Québec, 17e-20e siècles. Québec, 1985 (à paraître en 1986). 
Jusqu'en 1815 , le pain de froment demeure vraisemblablement l'un des aliments de base des maskoutains. Néanmoins, les habitudes alimentaires de la population n'expliquent pas totalement cette forte domination du froment. La nature éminemment commercialisable du blé favorise grandement sa production. Les marchands ruraux l'acceptent d'autant plus volontiers comme monnaie d'échange dans leurs transactions commerciales avec les paysans qu'ils peuvent en écouler assez régulièrement les surplus locaux à l'extérieur de la région. De plus, les terres sablo-argileuses de la plaine maskoutaine conviennent parfaitement à la culture de cette céréale et la plupart des contemporains s'accordent sur les rendements très élevés du froment sur les terres récemment défrichées ${ }^{29}$.

Les familles paysannes ne négligent pas pour autant une foule d'autres produits agricoles plus strictement liés aux marchés locaux et, surtout, aux besoins domestiques de la famille et de l'exploitation: l'avoine, l'orge, les pois, le lin, le tabac. Au début du 19e siècle, la pomme de terre s'insère graduellement dans le système cultural. Le tubercule est d'abord cultivé au jardin avec les autres légumes. Puis, le paysan lui consacre un petit lopin avant de l'intégrer dans le cycle des grandes cultures de l'exploitation. Cependant, avant 1815, l'intégration des pommes de terre dans le cycle des grandes cultures demeure plutôt marginale dans la plaine maskoutaine.

Par ailleurs, le manque de fourrage constitue l'une des principales carences de la production agricole. Ce problème ne se pose toutefois pas avec la même acuité dans toutes les exploitations paysannes. A cet égard, les inventaires après décès de certains cultivateurs recensent d'imposantes quantités de foin et de pailles diverses pour nourrir et soigner convenablement leurs cheptels ${ }^{30}$. Néanmoins, la production du foin, principale composante du fourrage, s'effectue toujours sur la base des procédés traditionnels, c'est-à-dire en comptant sur la productivité de prairies naturelles ou en convertissant en prairies certains lopins de l'exploitation soustraits, de cette manière, à la rotation des cultures ${ }^{31}$.

29 Voir les divers témoignages de l'enquête sur l'agriculture au Bas-Canada, Journaux de la Chambre d'Assemblée du Bas-Canada (1816): app. E.

30 C'est le cas notamment de la communauté Louis Plamondon et Marie-Barbe Saint-Marc dont l'inventaire des biens, à l'automne 1806, recense 6200 bottes de foin, 40 voyages de peza et 30 voyages de paille courte et longue d'une valeur de 2112 livres. Les Plamondon accaparent toutefois près de $40 \%$ de la valeur totale des fourrages recensés dans l'ensemble des 147 inventaires paysans de la décennie 1805 à 1814. A noter, leur cheptel comprend, entre autres, 7 chevaux, 68 bovins et 177 moutons. ANQ-M, minute P.-A. Gauthier, no 596, le 20 octobre 1806.

«...à charge en outre de 400 bottes de foin telles que les produise le lopin de $11 / 2$ sur 3 arpents dans le meilleur de la terre, lequel sera laissé tous les ans pour prairie...» [ANQ-M, minute J.-M. Mondelet, no 457, le 31 octobre 1796: donation de J.-B. Valin et M. Plamondon à J. Valin, fils] «...si le preneur venait à laisser un terrain pour faire une prairie, il aura droit d'enlever, à l'échéance de son bail, la moitié du foin qu'elle produira...» [ANQ-M, minute L. Picard, no 2053, le 4 août 1804: bail à ferme pour neuf années de G. Lapré dit Petit à L. Marcorelle] «...ne pourra labourer et ensemencer le terrain qui a été mis en prairie depuis plusieurs années...» [ANQ-M, minute L. Picard, no 2534, le 6 septembre 1806: bail à ferme pour trois années de J.-B. Vacher à T. Godin fils]. 
Or, pour les partisans d'une agriculture nouvelle, l'introduction des cultures fourragères dans la rotation même des cultures constitue l'élément de base d'une transformation du système cultural traditionnel.

La plaine maskoutaine est un pays d'assolement biennal. Contrairement aux critiques abusives de certains historiens, les paysans bascanadiens n'ignoraient pas le principe de l'assolement des terres ${ }^{32}$. Les baux ruraux sont témoins de l'application stricte de l'alternance des cultures et des jachères ${ }^{33}$. C'est ce système traditionnel d'assolement des terres que dénoncent d'ailleurs les disciples locaux de la nouvelle agriculture à l'anglaise ${ }^{34}$. Ces derniers proposent aux paysans l'adoption de systèmes de rotation de cultures plus complexes, à soles multiples, et l'élimination de la jachère dite morte. Selon eux, l'introduction de ces nouveaux systèmes de culture donnerait aux terres bascanadiennes une productivité comparable à celle des régions les plus riches du Royaume-Uni. De prime abord, les énoncés de ces critiques contemporains peuvent apparaître séduisants: suppression de la jachère, utilisation maximale de la terre, accroissement de la production fourragère et des rendements céréaliers. Cependant, à court terme, les paysans peuvent-ils assumer les coûts inhérents à l'adoption d'un système de culture où les emblavures céréalières sont proportionnellement réduites? Par ailleurs, les modèles d'exploitation envisagés, qui couvrent habituellement de grandes superficies et requièrent une abondante main-d'oeuvre salariée, correspondent davantage à la réalité économique et sociale de l'Angleterre qu'à celle du Bas-Canada ${ }^{35}$. On peut donc

32 «Au lieu de procéder à une rotation annuelle, la majorité des paysans cultivaient le blé, une céréale exigeante entre toutes, sur la même parcelle jusqu'à l'épuisement, recommençant ensuite le même processus sur la seconde et ensuite sur la troisième de l'espace défriché.» $\mathrm{F}$. Ouellet, Histoire économique et sociale du Québec, 1760-1850 (Montréal, Fides, 1971), 82.

33 Les seules dérogations à l'assolement des terres interviennent sur des parcelles nouvellement défrichées, mais pour un nombre d'années strictement limité. Par exemple, en 1803, JeanBaptiste Bourgeois permet à son fermier, Louis-Jérôme Jarret dit Beauregard, «à l'égard de la terre neuve qu'il pourra faire, il la sèmera les deux premières années qu'elle sera faite et ensuite ne la sèmera que tous les deux ans» (ANQ-M, minute L. Picard, no 1614, le 18 juin 1803; bail à ferme pour neuf années de J.-B. Bourgeois et L.-J. Jarret dit Beauregard]. Il s'agit en quelque sorte d'un avantage donné au fermier pour le dédommager de travaux de défrichement éventuels. Si la terre neuve n'était pas le résultat d'un sur-travail du fermier, le propriétaire ne se montrerait peut-être pas aussi ouvert à la perspective d'une déviation dans la pratique de l'assolement biennal. En 1809, Michel Bonnet précise à son métayer Jean-Baptiste Lhéro «d'ensemencer la moitié de la terre chaque année» et d'exploiter «dès le printemps prochain, la terre faite qui n'a pas encore été levée et la cultiver comme le reste de la terre tous les deux ans» [ANQ-M, minute L. Picard, no 2941, le 24 juillet 1809: bail à métayage pour trois années de M. Bonnet à J.-B. Lhéro].

34 «Le système généralement adopté dans ce pays est du grain une année et du foin l'autre année; mais on pourrait mieux dire que la terre ne fait rien, suivant cet arrangement, car au lieu $\mathrm{du}$ foin elle ne produit que quelques mauvaises herbes». $J C A B C, 1816$, App. E, témoignage de D. Anderson. Encore vers 1850, «les Canadiens français divisent les terres en deux parties, semées alternativement sans semer de graines de foin sur la partie en pâturage». AJALPC, 1850, App. TT, témoignage de W. Evans.

A ce propos, nous rejoignons Allan Greer, «For example, one expert witness testifying before the agriculture committee of the House of Assembly in 1816 suggested as the ideal system of cultivation a ten-course crop rotation scheme suitable for a 750-acre farm with fifteen workers, twenty-five horses, eleven ploughs, and so on (...) Obviously a habitant family could not afford to experiment in this way with crops it needed for food, nor could it procure the land, capital, and labour needed for some of the more elaborate systems of cultivation». A. Greer, op. cit., 216217. 
à juste titre se questionner sur la pertinence des modèles proposés par rapport aux structures sociales existantes dans les campagnes bas-canadiennes.

Le manque d'engrais, dont le fumier animal demeure la source presque exclusive, est certainement l'un des problèmes majeurs de l'agriculture maskoutaine. Néanmoins, il serait discutable de mettre en cause la mentalité paysanne à ce propos. La mauvaise fumure des terres est directement liée à l'insuffisance du cheptel dont la responsabilité incombe tant au manque de capitaux qu'à la carence des fourrages. Face à la pénurie, la solution de la plupart des cultivateurs est de rationaliser au mieux l'épandage du fumier. Dans la majorité des exploitations paysannes, le fumier sert presque exclusivement à enrichir le potager familial et certains lopins de terre affectés à des cultures secondaires particulièrement exigeantes telles le lin, le tabac et les pommes de terre. Dans les plus riches exploitations où le bétail est plus nombreux et mieux nourri, le cultivateur emploie une bonne partie des fumiers pour engraisser ses prairies. Enfin, le cas échéant, il épand les excédents sur la jachère. L'efficacité de cette dernière opération demeure certainement très limitée ${ }^{36}$.

Parmi le cycle des travaux champêtres, le labourage constitue l'activité première de l'agriculture ancienne ${ }^{37}$. L'étude du labourage ne se limite pas à des questions d'ordre technique. Elle permet également de rejoindre, par la possession ou non du train de labour, l'un des fondements matériels de la hiérarchie paysanne. Jusqu'au début du 19e siècle, les cultivateurs canadiens emploient presque exclusivement la lourde charrue à rouelles, munie d'un avant-train. L'emploi de cette charrue requiert, surtout dans les terres neuves et argileuses de la plaine maskoutaine, un attelage relativement puissant d'au moins deux boeufs jusqu'à quatre boeufs et deux chevaux pour un maximum d'efficacité ${ }^{38}$. Or, à Saint-Hyacinthe, moins de la moitié des familles paysannes possèdent une paire de boeufs de trait et moins du quart, deux paires de boeufs ou plus.

L'étude des autres travaux du cycle cultural, comme les semailles, le sarclage, la moisson et le battage des grains, de même que celle des divers travaux d'aménagement et d'entretien de l'exploitation, permet-

36 C'est d'ailleurs cette dernière opération qui est l'objet des critiques de plusieurs observateurs du 19e siècle qui soulignent les mauvaises techniques d'épandage et le manque de fumiers employés.

J. Meuvret, Le problème des subsistances à l'époque de Louis XIV. La production des céréales dans la France du XVIIe et du XVIIIe siècles (Paris, Mouton, 1977), 103.

38 En se référant aux premiers écrits agronomiques du $19 \mathrm{e}$ siècle, Louise Dechêne écrit, «On (la charrue canadienne) y attelle généralement quatre boeufs et, pour les faire avancer plus vite, un ou deux chevaux». L. Dechêne, "Observations sur l'agriculture du Bas-Canada au début du XIXe siècle», dans J. Goy et J.-P. Wallot, Évolution et éclatement du Monde rural. FranceQuébec XVIIe-XXe siècles (Montréal, Presses de l'Université de Montréal, 1986), 196. 
tent de saisir la lourdeur de la tâche du paysan dont l'accomplissement et le succès sont conditionnés par la courte durée de la saison végétative au Bas-Canada, par les exigences de chaque étape du cycle cultural et par la force de travail nécessaire à chacune de ces étapes. Dans plusieurs exploitations, en l'occurrence les plus productives, la récolte du blé parce qu'elle requiert toujours l'emploi de la faucille, commande nécessairement l'embauche d'une main-d'oeuvre saisonnière ${ }^{39}$. Certains chefs d'exploitation doivent également recourir à des travailleurs extérieurs au groupe domestique pour effectuer le battage des grains ${ }^{40}$. Cependant, l'existence d'une main-d'oeuvre agricole permanente ou semipermanente dans les campagnes maskoutaines d'avant 1815 apparait moins sûre. A ce propos, les sources disponibles à l'historien sont plutôt muettes. Néanmoins, la forte disproportion entre la capacité productive de certaines exploitations paysannes et le nombre de bras disponibles au sein de l'unité familiale conduit inévitablement à considérer l'hypothèse d'un marché relativement important de main-d'oeuvre agricole dans la société rurale. Par contre, la substitution d'une partie de la force de travail des petites exploitations paysannes au profit des plus grandes contre une rémunération souvent versée en nature ou contre des services essentiels comme la location d'un train de labour représente toutefois une alternative davantage compatible avec la réalité sociale maskoutaine d'avant 1815 où prédomine le groupe des propriétaires exploitants. L'étude de la répartition des moyens de production (terre, cheptel et instruments de travail) confirme par ailleurs la présence de fortes inégalités entre les travailleurs de la terre.

\section{5 - LA HIÉRARCHIE PAYSANNE: LES MOYENS DE PRODUCTION ET LES NIVEAUX DE FORTUNE}

\section{$A$ - Les moyens de production}

\section{1 - La terre}

Au tournant du $19 \mathrm{e}$ siècle, la terre maskoutaine appartient d'abord aux paysans. Entre 1795 et 1814 , les ménages paysans représentent environ $95 \%$ des propriétaires ruraux et ils accaparent plus de $90 \%$ du

39 L'opération est nettement plus rapide à la faux qu'à la faucille. Cependant, l'usage de la faucille, plus précise et plus maniable, évite l'égrènement des épis et préserve ainsi la productivité des champs céréaliers dont les rendements, dans l'économie paysanne ancienne, sont relativement faibles. Sur l'utilisation de la faucille pour les récoltes dans le Québec rural d'avant 1850, voir R.-L. Séguin, «L'équipement aratoire pré-machiniste aux XVIIe, XVIIIe et XIXe siècles» dans C. Galarneau et E. Lavoie, éd., France et Canada français du XVIe au XXe siècle. Colloque d' histoire comparée, 130.

40 Le battage des grains est un travail long et épuisant qui est normalement effectué par une équipe de batteurs au cours de l'hiver et du printemps. Certains inventaires paysans font mention des frais encourus pour le battage des grains de la récolte annuelle. Par exemple, en 1800, ceux de la communauté Pierre Fortin et Marie-Anne Végiard se montent à 213 livres, soit environ le vingtième de la récolte brute de l'exploitation familiale. ANQ-M, minute J. Papineau, no 3438, le 7 octobre 1802 . 
sol $^{41}$. Cependant, l'appartenance paysanne recouvre de multiples conditions socio-économiques. L'étude de la répartition de la propriété foncière permet de saisir partiellement l'hétérogénéité économique et sociale de la paysannerie maskoutaine. Les inventaires paysans montrent ainsi l'existence, dans une région «frontière» où les terres neuves demeurent encore abondantes, d'une proportion relativement importante de ménages paysans sans terre au début du 19e siècle (voir tableau 2). Entre 1795 et 1804 , cette catégorie de familles paysannes représente moins de $6 \%$ des inventaires après décès. Au cours de la décennie suivante, le pourcentage des paysans sans terre grimpe à près de $12 \%$. L'accroissement des paysans sans terre n'est d'ailleurs pas la seule manifestation d'une amorce de changement structurel dans la répartition des avoirs fonciers au sein de la paysannerie maskoutaine au début du 19e siècle. On observe également un mouvement de contraction des petites exploitations paysannes de 90 vers 60 arpents $^{42}$.

Jusqu'en 1815, les propriétés rurales de moins de 60 arpents sont toutefois limitées à une petite minorité de familles paysannes tandis que les avoirs fonciers d'une superficie inférieure à 30 arpents demeurent l'exception. Ces constatations ne sont d'ailleurs guère surprenantes dans un terroir relativement nouveau. D'autant plus, comme l'a déjà souligné Louis Michel, que dans les paroisses du centre comme dans celles de peuplement précoce, «se manifeste plus ou moins tôt une certaine rigidité des structures agraires $»^{43}$. Les paysans refusent habituellement de morceler leurs propriétés en deçà d'un certain seuil, de manière à conserver des exploitations agricoles dont la capacité de production est suffisante pour faire vivre une famille.

En deçà de 180 arpents, la majeure partie des avoirs fonciers représente une seule et même exploitation familiale avec maison et autres bâtiments de production. Entre 1795 et 1814, les terres de 60 à 179 arpents regroupent environ 70 à $75 \%$ des propriétaires d'origine paysanne et elles accaparent près de $60 \%$ du sol. La différenciation dans la superficie des avoirs fonciers ne remet pas en cause la persistance du modèle dominant de l'exploitation familiale.

Au-dessus de 1.80 arpents, les propriétés rurales sont normalement constituées d'au moins deux entités foncières distinctes. Par contre, cela ne correspond pas nécessairement au cumul par un même proprié-

41 Pour une étude détaillée de la répartition de la terre à Saint-Hyacinthe, voir C. Dessureault, «La propriété rurale et la paysannerie dans la plaine maskoutaine, 1795-1814», communication présentée au colloque d'histoire comparée des sociétés rurales de la France et du Québec, 17e-20e siècles, Québec, 1985 (à paraître en 1986).

42 A cet égard, ce mouvement de contraction des propriétés rurales a accompagné la politique seigneuriale de concéder principalement des tenures de 60 arpents à compter de 1805 .

${ }_{43}$ L. Michel, "Varennes et Verchères, des origines au milieu du XIXe siècle: état d'une enquête», dans J. Goy et J.-P. Wallot, éd., Évolution et éclatement du Monde rural. FranceQuébec, XVIIe-XXe siècles, 329. 
Tableau 2

Répartition des ménages paysans selon la superficie de leurs propriétés rurales

et ventilation de la superficie possédée par catégories de propriétaires, 1795-1814

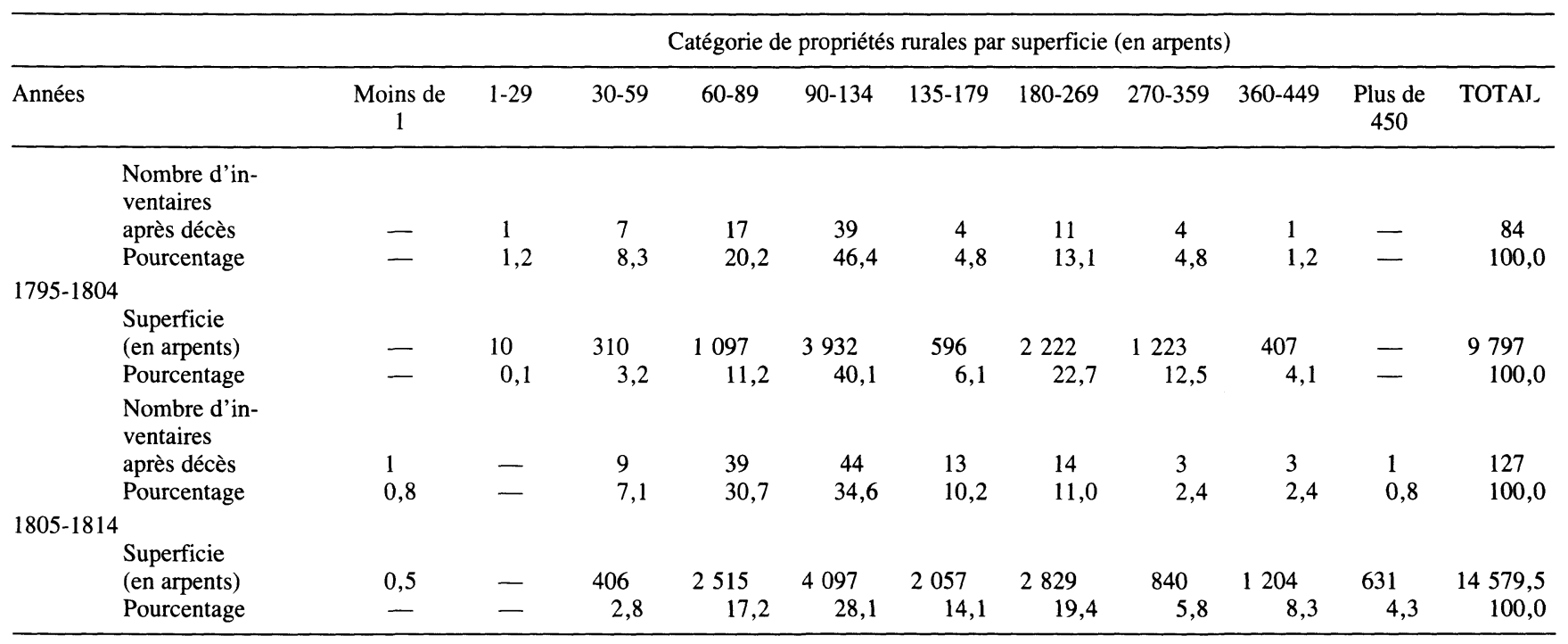

Source: Inventaires après décès. 
taire de plusieurs exploitations agricoles complètes avec maison, grange et autres bâtiments de production: le maximum recensé dans un inventaire de paysan maskoutain étant de trois exploitations agricoles complètes. Dans la majorité des avoirs fonciers de 180 arpents et plus, à la terre principale, foyer et fondement de l'exploitation familiale, se greffent une ou deux terres «en bois debout» ou peu défrichées.

Comme l'a souligné René Baehrel ${ }^{44}$, toutes les superficies ne sont pas comparables en valeur et en revenu. Dans la plaine maskoutaine, cette remarque conserve sa pertinence malgré l'unité apparente du paysage. Les écarts de valeur entre deux propriétés de même superficie peuvent être considérables. Certaines terres situées dans les nouveaux secteurs de peuplement ne valent pas le dixième de celles sises dans les terroirs plus anciens. A l'intérieur d'un même rang, voire entre deux voisins immédiats, la qualité inégale des bâtiments et l'ampleur respective des travaux d'aménagement et de culture du sol (fossés, cultures, labours) différencient nettement deux patrimoines de superficie comparable. Malheureusement, les sources disponibles permettent rarement une étude fine du terroir. Dans le cas maskoutain, nous avons pu évaluer une partie seulement des patrimoines fonciers dénombrés dans les inventaires paysans ${ }^{45}$. Cette démarche permet d'une part, de confronter valeur et superficie des propriétés rurales et d'autre part, d'affiner la hiérarchie des propriétaires fonciers au sein de la paysannerie (voir tableaux $3 a$ et $3 b$ ).

Le repérage de la valeur des propriétés rurales permet déjà de mieux saisir la diversité des conditions socio-économiques au sein de la petite paysannerie propriétaire. Par ailleurs, les liens entre la valeur et la superficie des propriétés rurales apparaissent trop faibles pour discerner, à partir des données sur la superficie, des signes évidents d'enrichissement ou d'appauvrissement au début du $19 \mathrm{e}$ siècle. L'échantillon des terres évaluées demeure d'ailleurs trop restreint pour soutenir une analyse vraiment fine de la répartition des avoirs fonciers au sein de la paysannerie. Même si les propriétés rurales de grande superficie apparaissent généralement se classer parmi les plus riches patrimoines, leur valeur repose souvent sur une seule de leurs composantes, la terre principale sur laquelle se trouvent la maison familiale et les bâtiments de production. La superficie de la terre s'avère donc un indice fragile

44 R. Baehrel, Une croissance: la Basse-Provence rurale (fin XVIe-1789) (Paris, SEPVEN, 1961), 2 .

Nos évaluations proviennent très rarement des inventaires paysans mêmes et des actes de partage des biens subséquents aux inventaires après décès. Nous avons dû chercher dans les greffes des notaires de Saint-Hyacinthe et des environs tous les contrats de vente de terres relatifs aux propriétés rurales maskoutaines. Seuls les contrats passés dans un intervalle de cinq ans antérieur ou postérieur à la dissolution d'une communauté ont été retenus afin de minimiser les écarts entre la valeur d'une terre au moment de l'inventaire et celle au moment de la transaction. De cette manière, nous avons retracé 36 des 84 patrimoines paysans de la décennie 1795 à 1804 et seulement 39 des 126 patrimoines paysans de la décennie 1805 à 1814 . 
Tableau 3A

Distribution des patrimoines fonciers paysans selon leur valeur et leur superficie, 1795-1814

\begin{tabular}{|c|c|c|c|c|c|c|c|}
\hline \multirow{2}{*}{$\begin{array}{l}\text { Superficie des } \\
\text { patrimoines } \\
\text { fonciers } \\
\text { (arpents) }\end{array}$} & \multicolumn{7}{|c|}{ Valeur des patrimoines fonciers (en livres) } \\
\hline & $0-499$ & $500-999$ & $1000-1999$ & 2000-3999 & $4000-7999$ & 8000 et + & Total \\
\hline $0-29$ & 1 & & & & & & 1 \\
\hline $30-59$ & 2 & & 1 & & & & 3 \\
\hline $60-89$ & 3 & 3 & 1 & 2 & 1 & & 10 \\
\hline $90-134$ & 3 & 7 & 2 & 3 & 2 & 1 & 18 \\
\hline $135-179$ & & & & 2 & & & 2 \\
\hline $\begin{array}{l}180-269 \\
\text { plus de } 270\end{array}$ & & & 1 & & 2 & & $\begin{array}{r}3 \\
-\end{array}$ \\
\hline Total & 9 & 10 & 5 & 7 & 5 & 1 & 37 \\
\hline$\%$ & 24,4 & 27,0 & 13,5 & 18,9 & 13,5 & 2,7 & 100 \\
\hline
\end{tabular}

Tableau 3B

\begin{tabular}{|c|c|c|c|c|c|c|c|}
\hline \multirow{2}{*}{$\begin{array}{l}\text { Superficie des } \\
\text { patrimoines } \\
\text { fonciers } \\
\text { (arpents) }\end{array}$} & \multicolumn{7}{|c|}{ Valeur des patrimoines fonciers (en livres) } \\
\hline & $0-499$ & $500-999$ & 1000-1999 & 2000-3999 & $4000-7999$ & 8000 et + & Total \\
\hline $0-29$ & & & & & & & - \\
\hline $30-59$ & 1 & & 2 & 1 & & & 4 \\
\hline $60-89$ & 6 & 1 & 3 & 1 & 1 & & 12 \\
\hline $90-134$ & 6 & 1 & 4 & 2 & 3 & & 16 \\
\hline $135-179$ & & & 1 & 2 & & 2 & 5 \\
\hline $180-269$ & & & & & 1 & & 1 \\
\hline plus de 270 & & & & & & 1 & 1 \\
\hline Total & 13 & 2 & 10 & 6 & 5 & 3 & 39 \\
\hline$\%$ & 33,3 & 5,2 & 25,7 & 15,4 & 12,8 & 7,7 & 100 \\
\hline
\end{tabular}

Sources: Inventaires après décès et registres d'état civil.

de la valeur comparée des propriétés rurales. De plus, la seule propriété de la terre ne garantit pas l'indépendance et la prospérité de la famille paysanne, surtout dans un terroir de peuplement récent. Le défrichement de la terre et la culture du sol commandent l'emploi, sinon la possession, d'animaux de trait et d'outils de production. A défaut de déterminer la valeur de l'ensemble des propriétés rurales, ces deux catégories d'avoirs mobiliers liés à la production agricole s'avèrent des mesures plus fiables de la hiérarchie paysanne. 


\section{2 - Le cheptel}

Les données sur la composition du cheptel moyen des familles paysannes maskoutaines, de 1795 à 1814, accréditent partiellement les conclusions habituelles de l'historiographie sur la faiblesse relative de l'élevage dans l'agriculture bas-canadienne (voir tableau 4). D'après

Tableau 4

Composition du cheptel moyen des familles paysannes, 1795-1814

\begin{tabular}{ccccccc}
\hline & Chevaux & Bovins & Ovins & Porcs & Volailles & $\begin{array}{c}\text { Nombre total } \\
\text { d'inventaires }\end{array}$ \\
\hline $1795-1804$ & 1,8 & 5,1 & 5,1 & 3,6 & 10,1 & 86 \\
$1805-1814$ & 1,8 & 6,4 & 8,4 & 2,6 & 9,2 & 144 \\
\hline
\end{tabular}

Source: Inventaires après décès.

ces données, les paysans maskoutains possèdent presque exclusivement les animaux d'attelage nécessaires aux travaux de défrichement et de culture du sol, ainsi que les animaux d'élevage et de reproduction requis pour répondre aux besoins domestiques de base de la famille et de l'exploitation agricole. Un examen attentif de la répartition des cheptels au sein du groupe paysan infirme toutefois ce modèle globalisant de l'auto-subsistance (voir tableaux 5, 6a et 6b).

\section{Tableau 5}

Distribution des ménages paysans maskoutains selon la valeur du cheptel vif et la possession de paires de boeufs de trait, 1795-1814

\begin{tabular}{|c|c|c|c|c|}
\hline \multirow{2}{*}{$\begin{array}{l}\text { Valeur du cheptel } \\
\quad \text { (en livres) }\end{array}$} & \multirow{2}{*}{$\begin{array}{c}\text { Nombre } \\
\text { de ménages }\end{array}$} & \multicolumn{3}{|c|}{ Nombre de ménages possédant: } \\
\hline & & $\begin{array}{l}1 \text { pre de } \\
\text { boeufs }\end{array}$ & $\begin{array}{c}2 \text { pres de } \\
\text { boeufs }\end{array}$ & $\begin{array}{c}3 \text { pres de } \\
\text { boeufs et plus }\end{array}$ \\
\hline Indéfini pauvre* & 6 & & & \\
\hline Nil & 12 & & & \\
\hline $1-100$ & 34 & 2 & & \\
\hline $101-250$ & 71 & 14 & & \\
\hline $251-500$ & 65 & 32 & 3 & 2 \\
\hline $501-950$ & 36 & 17 & 13 & 1 \\
\hline plus de 950 & 12 & 2 & 5 & 5 \\
\hline TOTAL & 236 & 67 & 21 & 8 \\
\hline
\end{tabular}

* Procès-verbaux de carence dans lesquels il n'y a pas la description et l'évaluation des biens mobiliers du ménage.

Source: Inventaires après décès. 
Tableau 6A

Répartition des ménages paysans maskoutains

selon le nombre d'animaux possédés

pour chacune des espèces animales, 1795-1804

\begin{tabular}{|c|c|c|c|c|c|c|c|}
\hline $\begin{array}{l}\text { A) BOVINS } \\
\text { Nombre d'inventaires }\end{array}$ & $\begin{array}{l}\text { Aucun } \\
11\end{array}$ & $\begin{array}{l}1-5 \\
41\end{array}$ & $\begin{array}{l}6-10 \\
21\end{array}$ & $\begin{array}{l}11-15 \\
11\end{array}$ & $\begin{array}{l}16-20 \\
2\end{array}$ & Plus de 20 & $\begin{array}{c}\text { Nombre total } \\
\text { d'inventaires* } \\
86\end{array}$ \\
\hline A') BOEUFS ADULTES & Aucun & 1 & $2-3$ & $4-5$ & $6-7$ & Plus de 8 & \\
\hline Nombre d'inventaires & 47 & 3 & 24 & 8 & 4 & & 86 \\
\hline B) CAVALES & Aucun & 1 & 2 & 3 & 4 & Plus de 5 & \\
\hline Nombre d'inventaires & 14 & 28 & 29 & 11 & 5 & 1 & 86 \\
\hline B') CHEVAUX ADULTES & Aucun & 1 & 2 & 3 & 4 & Plus de 5 & \\
\hline Nombre d'inventaires & 15 & 36 & 28 & 8 & 1 & & 86 \\
\hline C) OVINS & Aucun & $1-5$ & $6-10$ & $11-15$ & $16-20$ & Plus de 20 & \\
\hline Nombre d'inventaires & 31 & 21 & 20 & 7 & 5 & 2 & 86 \\
\hline D) PORCINS & Aucun & $1-3$ & $4-6$ & $7-9$ & $10-12$ & Plus de 12 & \\
\hline Nombre d'inventaires & 16 & 30 & 29 & 9 & 2 & & 86 \\
\hline E) VOLAILLES & Aucun & $1-10$ & $11-20$ & $21-30$ & $31-40$ & Plus de 40 & \\
\hline Nombre d'inventaires & 37 & 17 & 19 & 11 & 2 & & 86 \\
\hline
\end{tabular}

Source: Inventaires après décès. 
Tableau 6B

Répartition des ménages paysans maskoutains

selon le nombre d'animaux possédés

pour chacune des espèces animales, 1805-1814

\begin{tabular}{|c|c|c|c|c|c|c|c|}
\hline $\begin{array}{l}\text { A) BOVINS } \\
\text { Nombre d'inventaires }\end{array}$ & $\begin{array}{l}\text { Aucun } \\
13\end{array}$ & $\begin{array}{l}1-5 \\
71\end{array}$ & $\begin{array}{l}6-10 \\
35\end{array}$ & $\begin{array}{l}11-15 \\
14\end{array}$ & $\begin{array}{l}16-20 \\
8\end{array}$ & $\begin{array}{l}\text { Plus de } 20 \\
3\end{array}$ & $\begin{array}{c}\text { Nombre total } \\
\text { d'inventaires* } \\
144\end{array}$ \\
\hline A') BOEUFS ADULTES & Aucun & 1 & $2-3$ & $4-5$ & $6-7$ & Plus de 8 & \\
\hline Nombre d'inventaires & 77 & 7 & 43 & 13 & 2 & 2 & 144 \\
\hline B) CAVALES & Aucun & 1 & 2 & 3 & 4 & Plus de 5 & \\
\hline Nombre d'inventaires & 22 & 50 & 38 & 22 & 8 & 6 & 144 \\
\hline B') CHEVAUX ADULTES & Aucun & 1 & 2 & 3 & 4 & Plus de 5 & \\
\hline Nombre d'inventaires & 24 & 62 & 42 & 11 & 4 & 2 & 144 \\
\hline C) OVINS & Aucun & $1-5$ & $6-10$ & $11-15$ & $16-20$ & Plus de 20 & \\
\hline Nombre d'inventaires & 41 & 34 & 32 & 17 & 6 & 14 & 144 \\
\hline D) PORCINS & Aucun & $1-3$ & $4-6$ & $7-9$ & $10-12$ & Plus de 12 & \\
\hline Nombre d'inventaires & 25 & 80 & 29 & 7 & 3 & & 144 \\
\hline E) VOLAILLES & Aucun & $1-10$ & $11-20$ & $21-30$ & $31-40$ & Plus de 40 & \\
\hline Nombre d'inventaires & 63 & 30 & 28 & 15 & 3 & 5 & 144 \\
\hline
\end{tabular}

* 3 procès-verbaux de carence

Source: Inventaires après décès. 
Élément plutôt surprenant dans une économie paysanne dont l'objectif primordial serait, selon certains, l'auto-subsistance: aucune espèce animale n'est commune à toutes les familles paysannes. Les animaux domestiques les plus répandus sont dans l'ordre: la vache, le cheval et le porc. La possession d'une ou deux vaches laitières n'est pas généralisée dans la paysannerie maskoutaine. Entre 1795 et 1814, environ $10 \%$ des familles paysannes ne dénombrent aucun bovin, plus de $15 \%$ aucune vache. Par ailleurs, environ le sixième des ménages ne dispose pas d'un cheval adulte leur assurant une relative autonomie pour les travaux de défrichement et de charroi. La même proportion de ménages ne compte aucun porc qui les aiderait à équilibrer, à même leurs propres ressources, des rations alimentaires certainement pauvres en viande et en gras s'ils n'ont pas recours au marché local. Plus nombreuses encore sont les familles paysannes maskoutaines qui ne peuvent se fier sur leur propre élevage d'ovins et de volailles pour se procurer certains produits de consommation domestique comme la laine, les plumes d'oie, les oeufs et les chapons.

Dans la seconde décennie, on observe un accroissement du cheptel moyen des familles paysannes de Saint-Hyacinthe lié, en bonne partie, au progrès des défrichements et au développement du terroir agricole. Au bas de l'échelle davantage de familles possèdent au moins une vache laitière et l'élevage ovin apparaît désormais accessible à un plus grand nombre de cultivateurs. Mais, au cours de la même période, il y a une réduction sensible des cheptels moyens et, surtout, un renforcement important des gros élevages ovins et bovins: témoin d'une différenciation accrue au sein du groupe paysan. D'une décennie à l'autre, certains traits demeurent fondamentaux telle la division presque moitié-moitié entre les paysans qui disposent et ceux qui ne disposent pas de leur propre paire de boeufs de trait. De plus, cette division recoupe sensiblement celle de la valeur des cheptels au-dessus et en-dessous de 250 livres.

$\mathrm{Au}$ sommet de la hiérarchie paysanne se distingue une minorité d'environ $20 \%$ des ménages. Ces familles sont fort bien pourvues tant en animaux de travail qu'en animaux d'élevage et de reproduction. Ces cultivateurs possèdent au moins deux chevaux adultes. Ils élèvent également plus d'une dizaine de bovins et une vingtaine d'ovins, de même que des porcs et des volailles en quantité suffisante pour répondre aux besoins domestiques de la famille. Ils disposent normalement de leur propre paire de boeufs en âge de servir adéquatement aux travaux de labour et souvent plus de deux paires au fur et à mesure que s'accroît la valeur du cheptel. La plupart des autres familles ne sont pas totalement démunies. Mais nombreuses sont celles ne disposant ni d'un train de labour adéquat, ni d'un élevage suffisamment diversifié pour répondre aux principaux besoins de consommation domestique. 
Au début du $19 \mathrm{e}$ siècle, la dynamique du marché conditionne le développement de certains secteurs de l'élevage tels les ovins où la forte croissance du troupeau bénéficie surtout à une petite minorité de cultivateurs. Par contre, l'élevage des porcs et celui des volailles répondent davantage aux objectifs de l'auto-consommation. Néanmoins, la fonction première de l'élevage dans l'agriculture maskoutaine demeure toujours d'être un complément à la production agricole. En ce sens, les principales exploitations paysannes se caractérisent d'abord par l'importance de leur train de labour.

\section{3 - Les outils de production}

L'étude des instruments de travail complète l'analyse des moyens de production. Elle révèle d'abord la faible valeur marchande de l'équipement agricole comparativement au cheptel vif (voir tableau 7). L'en-

TABLEAU 7

Distribution des ménages paysans maskoutains selon la valeur des biens de production mobiliers, 1795-1814

\begin{tabular}{cc}
\hline $\begin{array}{c}\text { Valeur des } \\
\text { biens de production } \\
\text { (en livres) }\end{array}$ & $\begin{array}{c}\text { Nombre de } \\
\text { ménages }\end{array}$ \\
\hline Indéfini pauvre* & 6 \\
$0-50$ & 65 \\
$51-1.00$ & 70 \\
$101-1.50$ & 51 \\
$151-200$ & 19 \\
$210-250$ & 11 \\
Plus de 250 & 14 \\
TOTAL & 236 \\
\hline
\end{tabular}

* Procès-verbaux de carence dans lesquels il n'y a pas la description et l'évaluation des biens mobiliers du ménage.

Source: Inventaires après décès.

treprise paysanne investit autant, sinon davantage, dans la force de travail animale que dans le matériel de production: donnée typique d'une agriculture pré-machiniste. Par ailleurs, à l'instar du cheptel, les instruments de travail se répartissent inégalement au sein de la communauté paysanne. Plusieurs familles ne possèdent ni charrue, ni véhicule de travail. Par contre, au sommet de la hiérarchie, l'équipement de production comprend une gamme variée d'outils tractés (charrues, herses), de véhicules de travail (grande charrette, petite charrette, tombereau, traînes), d'accessoires d'attelage et d'outils manuels de défrichement 
et de culture du sol. Ce sont les familles les mieux nanties en cheptel et en outillage agricole qui disposent souvent de l'éventail le plus complet d'instruments de travail liés aux activités secondaires tel le textile domestique.

\section{$B$ - Les niveaux de fortune}

L'étude des bases matérielles de la production révèle une société paysanne fortement inégalitaire. La hiérarchie paysanne se vérifie également dans la distribution des fortunes mobilières (voir tableaux 8A et $8 B$ ). La richesse d'un ménage ne constitue pas le seul facteur de son rang social. Elle demeure toutefois le meilleur indice des inégalités économiques dans la communauté locale. Néanmoins, l'analyse ne doit pas se limiter au seul niveau de fortune. Il faut également considérer la composition de cette fortune afin de mieux saisir la signification réelle de tel ou tel niveau de richesse dans la structure sociale. A ce sujet, les particularités des fortunes paysannes supérieures apparaissent nettement: l'importance relative des stocks et des créances; le poids de moins en moins lourd des dettes par rapport aux avoirs mobiliers et fonciers. De plus, la part de moins en moins élevée des biens de consommation dans les actifs mobiliers au fur et à mesure de l'accroissement du niveau de fortune contredit l'image d'une paysannerie abusivement sensible au luxe et aux dépenses somptuaire ${ }^{46}$. La recherche du confort et du luxe dans les couches supérieures de la paysannerie, dont la nomenclature et la valeur réelle des biens de consommation seraient témoins, n'est pas nécessairement contradictoire avec l'accumulation de richesses productives, ni surtout avec le développement d'une agriculture de plus en plus axée vers le marché. La perspective d'un niveau de vie supérieur peut même s'avérer un stimulant efficace à l'accroissement des facteurs de production nécessaires pour acquérir le confort matériel et le statut social $^{47}$.

La hiérarchie paysanne maskoutaine n'apparaît pas comme le résultat, à long terme, d'un blocage malthusien. En 1815, les terres concédées représentent environ la moitié de la superficie totale de la seigneurie de Saint-Hyacinthe. Par ailleurs, la plupart des terres neuves alors disponibles s'insèrent dans l'un des meilleurs terroirs agricoles de la région de Montréal. La hiérarchie paysanne constitue plutôt une structure immanente de l'ancienne économie rurale de la vallée du SaintLaurent. Les données sur la répartition des avoirs mobiliers et fonciers révèlent tout de même une polarisation croissante de la société paysanne au début du $19 \mathrm{e}$ siècle.

46 F. Ouellet, «La mentalité et l'outillage économique du paysan canadien en 1760: à propos d'un document sur l'encan», Bulletin de recherches historiques, 62 (1956): 131-139.

47 E. J. Hobsbawn, «Capitalisme et agriculture: les réformateurs écossais au XVIIIe siècle», Annales ESC, 33,3 (mai-juin 1978): 580-601. 
TABLEAU 8A

Composition de la fortune paysanne selon le niveau des actifs mobiliers, 1795-1804*

\begin{tabular}{|c|c|c|c|c|c|c|c|c|c|c|c|}
\hline $\begin{array}{l}\text { Valeur des actifs } \\
\text { mobiliers } \\
\text { (en livres) }\end{array}$ & $\begin{array}{c}\text { Biens } \\
\text { de con- } \\
\text { sommation } \\
\%\end{array}$ & $\begin{array}{c}\text { Biens de } \\
\text { production } \\
\%\end{array}$ & $\begin{array}{c}\text { Cheptel } \\
\%\end{array}$ & $\begin{array}{c}\text { Stocks } \\
\%\end{array}$ & $\begin{array}{c}\text { Numéraire } \\
\%\end{array}$ & $\begin{array}{c}\text { Argen- } \\
\text { terie } \\
\%\end{array}$ & $\begin{array}{c}\text { Créances } \\
\%\end{array}$ & $\begin{array}{c}\text { Actifs } \\
\text { mobiliers } \\
\%\end{array}$ & $\begin{array}{c}\text { Passifs } \\
\%\end{array}$ & $\begin{array}{l}\text { Superficie } \\
\text { des terres } \\
\text { (en arpents) }\end{array}$ & $\begin{array}{c}\text { Nombre } \\
\text { total } \\
\text { d'I.A.D. }\end{array}$ \\
\hline $0-250$ & 33,8 & 9,5 & 19,1 & 10,7 & 6,5 & & 22,3 & 100,0 & 246,9 & 74,1 & 11 \\
\hline $251-500$ & 26,7 & 18,4 & 42,0 & 11,1 & & & 1,8 & 100,0 & 174,5 & 80,8 & 16 \\
\hline $501-10000$ & 25,4 & 15,4 & 34,2 & 13,4 & 0,7 & & 10,9 & 100,0 & 110,4 & 110,7 & 25 \\
\hline $1001-2000$ & 16,4 & 8,3 & 31,2 & 29,9 & 1,4 & & 12,8 & 100,0 & 60,0 & 120,2 & 22 \\
\hline $2001-4000$ & 13,2 & 6,2 & 26,5 & 24,5 & 2,0 & 0,3 & 27,3 & 100,0 & 24,7 & 156,9 & 8 \\
\hline $4001-8000$ & 11,1 & 5,5 & 14,5 & 32,9 & 6,2 & 0,1 & 29,7 & 100,0 & 22,1 & 185,0 & 4 \\
\hline Moyenne & 17,3 & 9,0 & 27,2 & 25,0 & 2,6 & 0,1 & 18,8 & 100,0 & 62,5 & 110,7 & 86 \\
\hline
\end{tabular}

* 6 procès-verbaux de carence dans lesquels il n'y avait pas la description et l'évaluation des biens mobiliers et fonciers n'ont pas été retenus pour ce tableau. Ces documents concernent néanmoins des ménages au bas de l'échelle sociale de la communauté paysanne maskoutaine.

\section{TABLEAU 8B}

Composition de la fortune paysanne selon le niveau des actifs mobiliers, 1805-1814*

\begin{tabular}{|c|c|c|c|c|c|c|c|c|c|c|c|}
\hline $\begin{array}{l}\text { Valeur des actifs } \\
\text { mobiliers } \\
\text { (en livres) }\end{array}$ & $\begin{array}{c}\text { Biens } \\
\text { de con- } \\
\text { sommation } \\
\%\end{array}$ & $\begin{array}{c}\text { Biens de } \\
\text { production } \\
\%\end{array}$ & $\begin{array}{c}\text { Cheptel } \\
\%\end{array}$ & $\begin{array}{c}\text { Stocks } \\
\%\end{array}$ & $\begin{array}{c}\text { Numéraire } \\
\%\end{array}$ & $\begin{array}{l}\text { Argen- } \\
\text { terie } \\
\%\end{array}$ & $\begin{array}{c}\text { Créances } \\
\%\end{array}$ & $\begin{array}{c}\text { Actifs } \\
\text { mobiliers } \\
\%\end{array}$ & $\begin{array}{c}\text { Passifs } \\
\%\end{array}$ & $\begin{array}{l}\text { Superficie } \\
\text { des terres } \\
\text { (en arpents) }\end{array}$ & $\begin{array}{c}\text { Nombre } \\
\text { Total } \\
\text { d'I.A.D. }\end{array}$ \\
\hline $0-250$ & 30,0 & 15,7 & 39,1 & 8,5 & 0,1 & & 6,0 & 100,0 & 571,0 & 64 & 24 \\
\hline $251-500$ & 29,4 & 14,9 & 39,0 & 11,3 & 0,2 & & 5,2 & 100,0 & 217,9 & 64,2 & 29 \\
\hline $501-1000$ & 22,6 & 12,0 & 41,0 & 13,8 & 1,1 & & 9,5 & 100,0 & 87,6 & 80,3 & 41 \\
\hline $1001-2000$ & 19,9 & 9,7 & 33,0 & 31,2 & 0,6 & & 5,6 & 100,0 & 57,6 & 113,8 & 28 \\
\hline $2001-4000$ & 12,7 & 7,8 & 29,2 & 36,2 & 0,9 & & 13,2 & 100,0 & 37,8 & 173,1 & 14 \\
\hline $4001-8000$ & 10,9 & 4,4 & 20,6 & 33,6 & 14,5 & & 16,0 & 100,0 & 11,3 & 258,2 & 6 \\
\hline Plus de 8000 & 10,7 & 2,6 & 16,8 & 31,0 & 1,9 & & 37,0 & 100,0 & 4,6 & 458 & 2 \\
\hline Moyenne & 16,3 & 7,8 & 28,5 & 28,1 & 3,3 & & 16,0 & 100,0 & 58,3 & 102,2 & 144 \\
\hline
\end{tabular}

Sources: Inventaires après décès. 
La crise ponctuelle de 1803-1804 n'est certainement pas étrangère à cette différenciation accrue au sein de la paysannerie maskoutaine. Elle apparaît directement responsable de l'accroissement du nombre de familles pauvres, voire démunies, dans la masse paysanne et de l'alourdissement de leur niveau d'endettement. Mais cette crise ne mène ni à l'effondrement du système de production, ni à un repli vers une agriculture d'auto-subsistance. Au contraire, le fort accroissement des actifs mobiliers et immobiliers de la couche supérieure de la paysannerie montre une agriculture de plus en plus articulée sur la production de surplus commercialisables. A moyen terme, les crises périodiques de l'ancienne économie rurale et la dynamique du marché favorisent le développement et la concentration des richesses productives au sein de la société paysanne.

Le cycle de la vie familiale est l'un des facteurs explicatifs des inégalités de fortune entre les ménages paysans ${ }^{48}$. La durée de la vie conjugale influe directement sur le niveau et sur la composition de la fortune paysanne (voir tableaux 9a et 9b). Dans l'ensemble de la communauté, on observe effectivement un processus d'accumulation des avoirs mobiliers et fonciers durant les quarante premières années de vie conjugale. Après quarante ans, la valeur des actifs mobiliers et la superficie des patrimoines fonciers diminuent fortement en raison des pratiques de redistribution des avoirs familiaux et de la retraite de la vie active des chefs de ménage. La superficie des avoirs fonciers des vieux ménages régresse même en-dessous de celle possédée par les jeunes ménages de moins de dix ans de vie conjugale tandis que dans la plupart des catégories de biens mobiliers, ce sont ces jeunes ménages qui enregistrent les actifs les moins considérables.

Néanmoins, le cycle de la vie familiale n'apparaît pas le facteur déterminant des inégalités économiques au sein de la paysannerie. A chaque stade de la vie conjugale, la répartition de la fortune mobilière apparaît plutôt inégalitaire (voir tableaux 10a et 10b). La distribution des avoirs fonciers au sein de chaque tranche de ménages s'inscrit sensiblement dans la même perspective (voir tableaux 11a et 11b). Mais, à défaut de données exhaustives sur la valeur des terres, les actifs mobiliers constituent un meilleur indice du niveau réel de la fortune paysanne.

La durée de la vie conjugale demeure tout de même un des éléments explicatifs des inégalités économiques au sein de la paysannerie. Au début du cycle de la vie conjugale, la fortune d'un ménage n'a

48 Pour une étude détaillée de l'influence du cycle de la vie familiale sur la composition et sur la répartition des fortunes paysannes, voir $\mathrm{C}$. Dessureault, «Niveaux de fortune paysans et cycle de la vie familiale. Le cas des paysans maskoutains au tournant du $19 \mathrm{e}$ siècle». Communication présentée lors d'un séminaire sur les inventaires et ventes de meubles au IXe Congrès international d'histoire économique, Berne, 1986 (à paraître). 
TABLEAU 9A

Composition de la fortune mobilière paysanne

selon la durée maximale de la vie conjugale de l'un ou l'autre

des conjoints des communautés inventoriées, 1795-1804

\begin{tabular}{|c|c|c|c|c|c|c|c|c|c|c|c|}
\hline $\begin{array}{l}\text { Intervalle entre } \\
\text { le } 1^{\mathrm{er}} \text { mariage et } \\
\text { l'inventaire }\end{array}$ & $\begin{array}{c}\text { Biens de } \\
\text { consomma- } \\
\text { tion } \\
\text { en livres }\end{array}$ & $\begin{array}{l}\text { Biens de } \\
\text { produc- } \\
\text { tion } \\
\text { en livres }\end{array}$ & $\begin{array}{l}\text { Cheptel } \\
\text { en livres }\end{array}$ & $\begin{array}{l}\text { Stocks } \\
\text { en livres }\end{array}$ & $\begin{array}{l}\text { Numéraire } \\
\text { en livres }\end{array}$ & $\begin{array}{l}\text { Argenterie } \\
\text { en livres }\end{array}$ & $\begin{array}{l}\text { Créances } \\
\text { en livres }\end{array}$ & $\begin{array}{c}\text { Actif̂s } \\
\text { mobiliers } \\
\text { en livres }\end{array}$ & $\begin{array}{l}\text { Passifs } \\
\text { en livres }\end{array}$ & $\begin{array}{l}\text { Superficie } \\
\text { des } \\
\text { terres } \\
\text { (en arpent) }\end{array}$ & $\begin{array}{l}\text { Nombre } \\
\text { total } \\
\text { d'I.A.D. }\end{array}$ \\
\hline $\begin{array}{l}0-9 \text { ans } \\
\text { indice }\end{array}$ & $\begin{array}{r}164 \\
79\end{array}$ & $\begin{array}{l}83 \\
77\end{array}$ & $\begin{array}{r}204 \\
63\end{array}$ & $\begin{array}{l}97 \\
33\end{array}$ & $\begin{array}{l}13 \\
42\end{array}$ & & $\begin{array}{r}175 \\
78\end{array}$ & $\begin{array}{r}736 \\
62\end{array}$ & $\begin{array}{r}636 \\
85\end{array}$ & $\begin{array}{l}93.4 \\
85\end{array}$ & 36 \\
\hline $\begin{array}{l}10-19 \text { ans } \\
\text { indice }\end{array}$ & $\begin{array}{l}236 \\
114\end{array}$ & $\begin{array}{l}113 \\
105\end{array}$ & $\begin{array}{l}366 \\
113\end{array}$ & $\begin{array}{l}533 \\
179\end{array}$ & $\begin{array}{r}43 \\
139\end{array}$ & & $\begin{array}{r}163 \\
73\end{array}$ & $\begin{array}{r}1454 \\
122\end{array}$ & $\begin{array}{l}783 \\
105\end{array}$ & $\begin{array}{c}102.7 \\
93\end{array}$ & 24 \\
\hline $\begin{array}{l}20-29 \text { ans } \\
\text { indice }\end{array}$ & $\begin{array}{l}208 \\
100\end{array}$ & $\begin{array}{l}142 \\
131\end{array}$ & $\begin{array}{l}443 \\
137\end{array}$ & $\begin{array}{l}371 \\
124\end{array}$ & $\begin{array}{r}7 \\
23\end{array}$ & $\begin{array}{r}3 \\
300\end{array}$ & $\begin{array}{r}194 \\
87\end{array}$ & $\begin{array}{r}1368 \\
115\end{array}$ & $\begin{array}{r}1025 \\
137\end{array}$ & $\begin{array}{l}144.6 \\
131\end{array}$ & 24 \\
\hline $\begin{array}{l}30-39 \text { ans } \\
\text { indice }\end{array}$ & $\begin{array}{l}418 \\
202\end{array}$ & $\begin{array}{l}116 \\
107\end{array}$ & $\begin{array}{l}362 \\
112\end{array}$ & $\begin{array}{r}225 \\
76\end{array}$ & $\begin{array}{l}309 \\
997\end{array}$ & $\begin{array}{r}5 \\
500\end{array}$ & $\begin{array}{r}1192 \\
532\end{array}$ & $\begin{array}{r}2627 \\
220\end{array}$ & $\begin{array}{r}207 \\
28\end{array}$ & $\begin{array}{l}95 \\
86\end{array}$ & 4 \\
\hline $\begin{array}{l}\text { Moyenne } \\
\text { indice }\end{array}$ & $\begin{array}{l}207 \\
100\end{array}$ & $\begin{array}{l}108 \\
100\end{array}$ & $\begin{array}{l}324 \\
100\end{array}$ & $\begin{array}{l}298 \\
100\end{array}$ & $\begin{array}{r}31 \\
100\end{array}$ & $\begin{array}{r}1 \\
100\end{array}$ & $\begin{array}{l}224 \\
100\end{array}$ & $\begin{array}{r}1193 \\
100\end{array}$ & $\begin{array}{l}746 \\
100\end{array}$ & $\begin{array}{l}110.0 \\
100\end{array}$ & $88 *$ \\
\hline
\end{tabular}

* Le calcul de la fortune mobilière a été fait sur la base de 86 inventaires tandis qu'un seul procès-verbal de carence est à la fois exclu du calcul de la fortune tant immobilière que mobilière.

Sources: Inventaires après décès et registres d'état civil. 
TABLEAU 9B

Composition de la fortune mobilière paysanne

selon la durée maximale de la vie conjugale de l'un ou l'autre

des conjoints des communautés inventoriées, 1805-1814

\begin{tabular}{|c|c|c|c|c|c|c|c|c|c|c|c|}
\hline $\begin{array}{l}\text { Intervalle entre } \\
\text { le } 1^{\mathrm{er}} \text { mariage } \\
\text { et l'inventaire }\end{array}$ & $\begin{array}{c}\text { Biens de } \\
\text { consomma- } \\
\text { tion } \\
\text { en livres }\end{array}$ & $\begin{array}{l}\text { Biens de } \\
\text { produc- } \\
\text { tion } \\
\text { en livres }\end{array}$ & $\begin{array}{l}\text { Cheptel } \\
\text { en livres }\end{array}$ & $\begin{array}{l}\text { Stocks } \\
\text { en livres }\end{array}$ & $\begin{array}{l}\text { Numéraire } \\
\text { en livres }\end{array}$ & $\begin{array}{l}\text { Argenterie } \\
\text { en livres }\end{array}$ & $\begin{array}{l}\text { Créances } \\
\text { en livres }\end{array}$ & $\begin{array}{c}\text { Actifs } \\
\text { mobiliers } \\
\text { en livres }\end{array}$ & $\begin{array}{l}\text { Passifs } \\
\text { en livres }\end{array}$ & $\begin{array}{c}\text { Superficie } \\
\text { des } \\
\text { terres } \\
\text { (en arpent) }\end{array}$ & $\begin{array}{c}\text { Nombre } \\
\text { total } \\
\text { d'I.A.D. }\end{array}$ \\
\hline $\begin{array}{l}0-9 \text { ans } \\
\text { indice }\end{array}$ & $\begin{array}{r}127 \\
62\end{array}$ & $\begin{array}{l}74 \\
75\end{array}$ & $\begin{array}{r}242 \\
67\end{array}$ & $\begin{array}{l}360 \\
101\end{array}$ & $\begin{array}{r}7 \\
17\end{array}$ & & $\begin{array}{l}43 \\
21\end{array}$ & $\begin{array}{r}853 \\
72\end{array}$ & $\begin{array}{r}548 \\
75\end{array}$ & $\begin{array}{l}77.6 \\
76\end{array}$ & 40 \\
\hline $\begin{array}{l}10-19 \text { ans } \\
\text { indice }\end{array}$ & $\begin{array}{r}184 \\
89\end{array}$ & $\begin{array}{l}97 \\
97\end{array}$ & $\begin{array}{r}330 \\
90\end{array}$ & $\begin{array}{r}319 \\
88\end{array}$ & $\begin{array}{r}5 \\
12\end{array}$ & & $\begin{array}{l}66 \\
33\end{array}$ & $\begin{array}{r}1001 \\
77\end{array}$ & $\begin{array}{l}890 \\
121\end{array}$ & $\begin{array}{l}112.7 \\
110\end{array}$ & 49 \\
\hline $\begin{array}{l}20-29 \text { ans } \\
\text { indice }\end{array}$ & $\begin{array}{l}299 \\
145\end{array}$ & $\begin{array}{l}113 \\
114\end{array}$ & $\begin{array}{l}506 \\
139\end{array}$ & $\begin{array}{l}395 \\
110\end{array}$ & $\begin{array}{l}28 \\
67\end{array}$ & & $\begin{array}{l}304 \\
150\end{array}$ & $\begin{array}{r}1645 \\
134\end{array}$ & $\begin{array}{l}896 \\
122\end{array}$ & $\begin{array}{l}116.8 \\
114\end{array}$ & 26 \\
\hline $\begin{array}{l}30-39 \text { ans } \\
\text { indice }\end{array}$ & $\begin{array}{l}282 \\
137\end{array}$ & $\begin{array}{l}138 \\
139\end{array}$ & $\begin{array}{l}519 \\
143\end{array}$ & $\begin{array}{l}458 \\
129\end{array}$ & $\begin{array}{l}208 \\
495\end{array}$ & & $\begin{array}{l}528 \\
260\end{array}$ & $\begin{array}{r}2133 \\
167\end{array}$ & $\begin{array}{r}587 \\
80\end{array}$ & $\begin{array}{l}119 \\
116\end{array}$ & 23 \\
\hline $\begin{array}{l}\text { Plus de } 40 \text { ans } \\
\text { indice }\end{array}$ & $\begin{array}{l}226 \\
110\end{array}$ & $\begin{array}{l}89 \\
90\end{array}$ & $\begin{array}{r}318 \\
88\end{array}$ & $\begin{array}{r}214 \\
60\end{array}$ & $\begin{array}{l}21 \\
50\end{array}$ & & $\begin{array}{l}257 \\
127\end{array}$ & $\begin{array}{r}1125 \\
88\end{array}$ & $\begin{array}{l}945 \\
129\end{array}$ & $\begin{array}{l}71.7 \\
70\end{array}$ & 9 \\
\hline $\begin{array}{l}\text { Moyenne } \\
\text { indice }\end{array}$ & $\begin{array}{l}207 \\
100\end{array}$ & $\begin{array}{r}99 \\
100\end{array}$ & $\begin{array}{l}363 \\
100\end{array}$ & $\begin{array}{l}358 \\
100\end{array}$ & $\begin{array}{r}42 \\
100\end{array}$ & & $\begin{array}{l}203 \\
100\end{array}$ & $\begin{array}{r}1272 \\
100\end{array}$ & $\begin{array}{l}734 \\
100\end{array}$ & $\begin{array}{l}102.2 \\
100\end{array}$ & $144^{*}$ \\
\hline
\end{tabular}

* Le calcul de la fortune mobilière et immobilière a été fait sur la base de 144 inventaires après décès; trois procès-verbaux de carence ont été exclus du calcu tant de la fortune mobilière qu'immobilière.

Sources: Inventaires après décès et registres d'état civil. 
TABLEAU 10A

Distribution des fortunes mobilières paysannes (actifs)

selon la durée maximale de vie conjugale de l'un

ou l'autre des conjoints de la comnıunauté dissoute 1795-1804

\begin{tabular}{|c|c|c|c|c|c|c|c|c|c|c|}
\hline \multirow{2}{*}{$\begin{array}{l}\text { Intervalle entre } \\
\text { le } 1^{\text {er }} \text { mariage } \\
\text { et l'I.A.D. }\end{array}$} & \multicolumn{9}{|c|}{ Valeur des fortunes mobilières (en livres) } & \multirow{2}{*}{$\begin{array}{c}\text { Nombre } \\
\text { total } \\
\text { d'I.A.D. }\end{array}$} \\
\hline & Ind. P. & Nil & $\begin{array}{c}1- \\
250\end{array}$ & $\begin{array}{l}251- \\
500\end{array}$ & $\begin{array}{l}501- \\
1000\end{array}$ & $\begin{array}{l}1001- \\
2000\end{array}$ & $\begin{array}{l}2001- \\
4000\end{array}$ & $\begin{array}{l}4001- \\
8000\end{array}$ & $\begin{array}{c}\text { Plus de } \\
8000\end{array}$ & \\
\hline $0-9$ ans & 1 & 1 & 5 & 10 & 13 & 4 & 2 & & & 36 \\
\hline $10-19$ ans & & & 3 & 3 & 6 & 9 & 1 & 2 & & 24 \\
\hline $20-29$ ans & 1 & & 2 & 2 & 5 & 9 & 4 & 1 & & 24 \\
\hline $30-39$ ans & & & & 1 & 1 & & 1 & 1 & & 4 \\
\hline $\begin{array}{l}\text { Indéfini } \\
\text { vieux }\end{array}$ & 1 & & & & & & & & & 1 \\
\hline Total & 3 & 1 & 10 & 16 & 25 & 22 & 8 & 4 & & 89 \\
\hline Pourcentage & $3,4 \%$ & $1,1 \%$ & $11,2 \%$ & $18,0 \%$ & $28,1 \%$ & $24,7 \%$ & $9,0 \%$ & $4,5 \%$ & & $100 \%$ \\
\hline
\end{tabular}

Sources: Inventaires après décès et registres d'état civil. 
TABLEAU 10B

Distribution des fortunes mobilières paysannes (actifs)

selon la durée maximale de vie conjugale de l'un

ou l'autre des conjoints de la communauté dissoute 1805-1814

\begin{tabular}{|c|c|c|c|c|c|c|c|c|c|c|}
\hline \multirow{2}{*}{$\begin{array}{l}\text { Intervalle entre } \\
\text { le } 1^{\mathrm{er}} \text { mariage } \\
\text { et l'I.A.D. }\end{array}$} & \multicolumn{9}{|c|}{ Valeur des fortunes mobilières (en livres) } & \multirow{2}{*}{$\begin{array}{c}\text { Nombre } \\
\text { total } \\
\text { d'I.A.D. }\end{array}$} \\
\hline & Ind. $P$. & Nil & $\begin{array}{c}1- \\
250\end{array}$ & $\begin{array}{l}251- \\
500\end{array}$ & $\begin{array}{l}501- \\
1000\end{array}$ & $\begin{array}{l}1001- \\
2000\end{array}$ & $\begin{array}{l}2001- \\
4000\end{array}$ & $\begin{array}{l}4001- \\
8000\end{array}$ & $\begin{array}{l}\text { Plus de } \\
8000\end{array}$ & \\
\hline $\begin{array}{l}0-9 \text { ans } \\
10-19 \text { ans } \\
20-29 \text { ans } \\
30-39 \text { ans } \\
\text { Plus de } 40 \text { ans } \\
\text { Ind. }\end{array}$ & $\begin{array}{l}1 \\
1 \\
1\end{array}$ & & $\begin{array}{l}7 \\
7 \\
5 \\
4 \\
1\end{array}$ & $\begin{array}{r}10 \\
10 \\
4 \\
3 \\
2\end{array}$ & $\begin{array}{r}11 \\
15 \\
6 \\
6 \\
3\end{array}$ & $\begin{array}{l}7 \\
9 \\
7 \\
4 \\
1\end{array}$ & $\begin{array}{l}4 \\
5 \\
2 \\
1 \\
2\end{array}$ & $\begin{array}{l}1 \\
2 \\
3\end{array}$ & $\begin{array}{l}1 \\
1\end{array}$ & $\begin{array}{r}40 \\
49 \\
26 \\
23 \\
9\end{array}$ \\
\hline $\begin{array}{l}\text { Total } \\
\text { Pourcentage }\end{array}$ & $\begin{array}{c}3 \\
2,0 \%\end{array}$ & & $\begin{array}{c}24 \\
16,3 \%\end{array}$ & $\begin{array}{c}29 \\
19,7 \%\end{array}$ & $\begin{array}{c}41 \\
28,0 \%\end{array}$ & $\begin{array}{c}28 \\
19,0 \%\end{array}$ & $\begin{array}{c}14 \\
9,5 \%\end{array}$ & $\begin{array}{c}6 \\
4,1 \%\end{array}$ & $\begin{array}{c}2 \\
1,4 \%\end{array}$ & $\begin{array}{c}147 \\
100 \%\end{array}$ \\
\hline
\end{tabular}

Sources: Inventaires après décès et registres d'état civil. 
TABLEAU 11A

Distribution des avoirs fonciers paysans (en superficie)

selon la durée maximale de la vie conjugale de l'un ou

l'autre des conjoints de la communauté dissoute, 1795-1804

\begin{tabular}{|c|c|c|c|c|c|c|c|c|c|c|c|c|}
\hline \multirow[b]{2}{*}{$\begin{array}{l}\text { Intervalle entre } \\
\text { le } 1^{\text {er }} \text { mariage et } \\
\text { l'inventaire }\end{array}$} & \multirow[b]{2}{*}{$\begin{array}{l}\text { Sans } \\
\text { propriété } \\
\text { rurale }\end{array}$} & \multicolumn{10}{|c|}{ Nombre de propriétaires par catégorie de terres en superficie (en arpents) } & \multirow[b]{2}{*}{$\begin{array}{l}\text { Nombre } \\
\text { total } \\
\text { d'I.A.D }\end{array}$} \\
\hline & & $\begin{array}{l}\text { Plus } \\
\text { de } 1\end{array}$ & $\begin{array}{l}1- \\
29\end{array}$ & $\begin{array}{c}30- \\
59\end{array}$ & $\begin{array}{c}60- \\
89\end{array}$ & $\begin{array}{l}90- \\
134\end{array}$ & $\begin{array}{l}135- \\
179\end{array}$ & $\begin{array}{l}180- \\
269\end{array}$ & $\begin{array}{l}270- \\
359\end{array}$ & $\begin{array}{c}360- \\
449\end{array}$ & $\begin{array}{c}\text { Plus de } \\
450\end{array}$ & \\
\hline $0-9$ ans & 2 & & & 4 & 9 & 15 & 2 & 4 & & & & 36 \\
\hline $10-19$ ans & 1 & & & 1 & 4 & 15 & & 2 & 1 & & & 24 \\
\hline $20-29$ ans & 1 & & 1 & 2 & 3 & 6 & 2 & 5 & 3 & 1 & & 24 \\
\hline $30-39$ ans & & & & & 1 & 2 & & & & & & 4 \\
\hline Indéfini & & & & & & 1 & & & & & & 1 \\
\hline Total & 5 & & 1 & 7 & 17 & 39 & 4 & 11 & 4 & 1 & & 89 \\
\hline Moyenne & 5,6 & & 1,1 & 7,9 & 19,1 & 43,8 & 4,5 & 12,4 & 4,5 & 1,1 & & 100 \\
\hline
\end{tabular}

Sources: Inventaires après décès et registres d’État civil. 


\section{TABLEAU 11B}

Distribution des avoirs fonciers paysans (en superficie)

selon la durée maximale de la vie conjugale de l'un ou

l'autre des conjoints de la communauté dissoute, 1805-1814

\begin{tabular}{|c|c|c|c|c|c|c|c|c|c|c|c|c|}
\hline \multirow[b]{2}{*}{$\begin{array}{l}\text { Intervalle entre } \\
\text { le }{ }^{\text {er }} \text { mariage et } \\
\text { l'inventaire }\end{array}$} & \multirow[b]{2}{*}{$\begin{array}{c}\text { Sans } \\
\text { propriété } \\
\text { rurale }\end{array}$} & \multicolumn{10}{|c|}{ Nombre de propriétaires par catégorie de terres en superficie (en arpents) } & \multirow[b]{2}{*}{$\begin{array}{c}\text { Nombre } \\
\text { total } \\
\text { d'I.A.D. }\end{array}$} \\
\hline & & $\begin{array}{l}\text { Plus } \\
\text { de } 1\end{array}$ & $\begin{array}{l}1- \\
29\end{array}$ & $\begin{array}{l}30- \\
59\end{array}$ & $\begin{array}{l}60- \\
89\end{array}$ & $\begin{array}{l}90- \\
134\end{array}$ & $\begin{array}{l}135- \\
179\end{array}$ & $\begin{array}{l}180- \\
269\end{array}$ & $\begin{array}{l}270- \\
359\end{array}$ & $\begin{array}{c}360- \\
449\end{array}$ & $\begin{array}{c}\text { Plus de } \\
450\end{array}$ & \\
\hline $0-9$ ans & 6 & & & 3 & 15 & 11 & 2 & 3 & & & & 40 \\
\hline $10-19$ ans & 2 & 1 & & 5 & 14 & 12 & 4 & 6 & 2 & 2 & & 48 \\
\hline $20-29$ ans & 3 & & & & 5 & 11 & 3 & 2 & & & 1 & 25 \\
\hline $30-39$ ans & 3 & & & 1 & 3 & 8 & 2 & 3 & 1 & 1 & & 22 \\
\hline Plus de 40 ans & 3 & & & & 2 & 2 & 2 & & & & & 9 \\
\hline Total & 17 & 1 & & 9 & 39 & 44 & 13 & 14 & 3 & 3 & 1 & $144^{*}$ \\
\hline Moyenne & 11,8 & 0,7 & & 6,2 & 27,1 & 30,6 & 9,0 & 9,7 & 2,1 & 2,1 & 0,7 & 100 \\
\hline
\end{tabular}

* 3 procès-verbaux de carence sans déclaration d'avoirs fonciers ont été exclus.

Sources: Inventaires après décès et registres d'État civil. 
évidemment pas atteint son niveau maximal. Ainsi, les jeunes ménages sont proportionnellement plus nombreux dans les catégories inférieures de la richesse mobilière. Cependant, après moins d'une dizaine d'années de mariage, certains ménages se classent déjà dans les échelons supérieurs de la fortune. Même dans un pays de peuplement récent et d'accès relativement large à la terre, les conditions de départ sont fortement conditionnées par l'appartenance sociale. L'accès aux fortunes supérieures au cours du cycle de vie ne constitue certainement pas une règle de fonctionnement de la société paysanne pré-industrielle. Le processus d'accumulation a d'autant plus de chances d'être effectif, sinon considérable, quand, dès le début de la vie active, les chefs de ménage disposent déjà des meilleurs atouts économiques et sociaux.

\section{CONCLUSION}

En général, l'historiographie identifie la paysannerie du Québec pré-industriel comme un groupe social distinct et relativement homogène. Les recherches sur la société rurale ont plutôt insisté, non sans raison, sur les rapports d'exploitation économique et sur les oppositions culturelles entre les paysans et les groupes dominants de la société rurale: clergé, seigneurs, marchands. Par contre, les auteurs n'ont guère scruté la composition interne de la paysannerie dont la cohésion leur apparaissait évidente dans des conditions historiques d'accès large à la terre.

Notre étude propose une vision très différente de la paysannerie et de la structure sociale du Québec pré-industriel. L'égalitarisme paysan dans l'ancienne société rurale de la vallée du Saint-Laurent est en grande partie un mythe frontiériste. Les paysans se distinguent non seulement par leurs niveaux de fortune, ce qui n'est pas négligeable, mais aussi dans leurs rapports à la production. La possession fort inégale des trains de labour apparaît un élément sinon un indice d'une division sociale typique des sociétés rurales d'Ancien Régime. Le rang social dans la communauté locale de tel ou tel ménage paysan et, à une échelle plus globale, la place de la paysannerie dans la structure sociale, n'est pas simple à déterminer. La stratification classique, par groupe socio-professionnel, résiste mal à cette hiérarchie interne de la paysannerie.

Notre recherche ne résout pas la question fondamentale du poids de la conjoncture économique dans le développement historique de la société rurale bas-canadienne. Elle conduit toutefois à refuser la conception selon laquelle les crises ponctuelles inhérentes aux anciennes économies rurales doivent nécessairement conduire à la cassure structurelle du système de production et entraver, de manière inéluctable, le développement des forces productives. Enfin, nous n'avons pas abordé le problème de la reproduction sociale au sein de la paysannerie. Néanmoins, le constat de la hiérarchie paysanne apporte certains éléments de réflexion aux études entreprises à ce propos. Dans l'ancienne société 
rurale de la vallée du Saint-Laurent, la paysannerie ne forme pas un groupe social homogène dont la stratégie de reproduction sociale repose sur les opportunités de redéploiement des familles dans l'espace et dont les rapports de parenté constituent l'essence des rapports sociaux ${ }^{49}$. La formation du prolétariat rural dans la première moitié du $19 \mathrm{e}$ siècle, de même que le recrutement du clergé, des marchands et d'autres notables au sein de la paysannerie, sont deux volets indissociables d'une reproduction sociale où tous les individus ne disposent pas, au départ, des mêmes atouts pour s'engager dans l'aventure commune de l'idéal américain.

49 A ce propos, voir le modèle de reproduction sociale de la paysannerie québécoise du $17 \mathrm{e}$ au milieu du $19 \mathrm{e}$ siècle proposé par G. Bouchard, "Les systèmes de transmission des avoirs familiaux et le cycle de la société rurale au Québec du XVIle au XXe siècles», Histoire sociale, 16,31 (mai 1983): 35-60. 